\title{
REFINED ANISOTROPIC K-TYPES AND SUPERCUSPIDAL REPRESENTATIONS
}

\author{
JEFFREY D. ADLER
}

\begin{abstract}
Let $F$ be a nonarchimedean local field, and $\mathrm{G}$ a connected reductive group defined over $F$. We classify the representations of $\mathbf{G}(F)$ that contain any anisotropic unrefined minimal $K$-type satisfying a certain tameness condition. We show that these representations are induced from compact (mod center) subgroups, and we construct corresponding refined minimal $K$-types.
\end{abstract}

\section{Introduction.}

Let $\mathbf{G}$ be any connected reductive group defined over a nonarchimedean local field $F$ of residual characteristic $p$. Under some tameness assumptions on $\mathbf{G}$, we construct families of positive-depth supercuspidal representations of $G=\mathbf{G}(F)$. In particular, we classify (§2.7) the representations of $G$ that contain any anisotropic unrefined minimal K-type (in the sense of MoyPrasad [28]) that satisfies a tameness condition. These representations are induced (§2.5), and from the inducing data (§2.6) we construct corresponding refined minimal K-types (§2.8), which are just types in the sense of BushnellKutzko [4].

One feature of this construction is that the resulting families of representations need not be associated to maximal anisotropic tori. Instead, they may arise from centralizers of singular anisotropic elements, or, depending on one's point of view, certain non-maximal anisotropic tori (those that have compact centralizers). Such "singular" supercuspidal representations are implicit in the work of Moy [24, 26] and Jabon [17] on $\mathrm{U}(2,1)$ and $\mathrm{GSp}_{4}$, but this is apparently the first general construction that produces them. (Another construction, using a different approach, is due to Kim [19].)

Otherwise, the families that arise here are analogous to those constructed by Carayol [5] for $\mathrm{GL}_{n}$, those that arise from generic characters in the Howe construction [13] for $\mathrm{GL}_{n}$, and those that arise from "cuspidal data of rank 1" (and sometimes higher rank) in the work of Morris on classical groups $[\mathbf{2 1}, \mathbf{2 2}, \mathbf{2 3}]$. They also include the families constructed by Gérardin [10] for Chevalley groups. 
Many of the methods that we use are analogous to those used by Howe in [14] and by Moy in [26]. However, some effort is required to make them work in a more general context. The first ingredient required is a set of filtrations, both on the parahoric subgroups and on the Lie algebra. This is provided by Moy and Prasad [27]. For every point $x$ in the Bruhat-Tits building $\mathcal{B}$ of $G$, Moy and Prasad define a parahoric subgroup $G_{x}$, a filtration $\left\{G_{x, r}\right\}_{r \geq 0}$ of $G_{x}$, and filtrations $\left\{\mathfrak{g}_{x, r}\right\}_{r \in \mathbb{R}}$ and $\left\{\mathfrak{g}_{x, r}^{*}\right\}_{r \in \mathbb{R}}$ of the Lie algebra $\mathfrak{g}$ and its dual $\mathfrak{g}^{*}$. In $\S 1.4$, we describe these filtrations (while renormalizing their indexing, following [30]) and show that they are compatible with Galois descent, a fact that we will use often. One consequence is that the filtrations (though not the parahoric subgroups) could have been defined in terms of split (rather than quasi-split) groups and Galois descent.

In order to pass from the Lie algebra to the group, we need a substitute for the exponential map. When $G=\mathrm{GL}_{n}$, one can use the map $X \longmapsto 1+X$; for symplectic, orthogonal, and unitary groups, one can use the Cayley transform. More generally, for each $x \in \mathcal{B}$ we define a non-canonical, filtrationpreserving, "mock exponential" function

$$
\varphi_{x}: \mathfrak{g}_{x, 0^{+}} \longrightarrow G_{x, 0^{+}}
$$

(a "+" on an index has the effect of adding a small positive number to it) having most of the useful properties of the map $X \longmapsto 1+X$. This is carried out in $\S \S 1.5-1.6$, using preliminary work in $\S \S 1.2-1.3$. One property that is lacking is $G$-invariance, but this is not necessary for the present application.

In the setup of Moy and Prasad [27], there is a correspondence between the characters of $G_{x, r} / G_{x, r^{+}}$and the cosets in $\mathfrak{g}_{x,-r}^{*} / \mathfrak{g}_{x,(-r)^{+}}^{*}$. A similar fact is true for characters of certain other subquotients of $G_{x}$, and the precise statement is given in $\S 1.7$.

Admissible representations of $G$ having positive depth should typically be associated to representations of certain other groups, which often arise as centralizer subgroups of $G$. In $\S 1.9$, we show that the Moy-Prasad filtrations and the mock exponential maps are compatible with restriction to such subgroups.

All of the constructions in $\S 1$ are independent of the choice of nonarchimedean local field $F$, reductive group $\mathbf{G}$, and point $x \in \mathcal{B}$. They should have wider uses than the ones to which we put them here.

Under some hypotheses on the residual characteristic $p$ of $F$, one can construct [2] a nondenegerate, symmetric, bilinear form $B$ on $\mathfrak{g}$, related to the Killing form, such that the corresponding identification of $\mathfrak{g}$ with its dual also identifies each $\mathfrak{g}_{x, r}$ with $\mathfrak{g}_{x, r}^{*}$. We state the necessary hypotheses in $\S 2.1$, and freely make this identification thereafter.

Our constructions work for certain unrefined minimal K-types, which we 
call "good." Roughly, a K-type is good if, over some tame field extension, it looks like it is contained in a principal series representation. The precise definition appears in $\S 2.2$. While the typical unrefined minimal K-type need not be good, there is reason to believe that, in the absence of wild ramification, all irreducible smooth representations of $G$ of positive depth contain good K-types. This is certainly the case for representations of $\mathrm{GL}_{n}[\mathbf{1 4}, \mathbf{1 5}, \mathbf{1 6}]$, $\mathrm{GSp}_{4}[\mathbf{2 6}], \mathrm{U}(2,1)[\mathbf{2 4}$, and division algebras [9]; the supercuspidals constructed by Gérardin [10] and (apparently) by Morris [21, 22, 23] and Kim [19]; and principal series representations, at least under some hypotheses on $p$ [32]. Thus, restricting our attention to good $\mathrm{K}$-types is probably no more serious than ignoring wild ramification.

For any representation $\rho$ of a compact open subgroup of $G$, one can consider the Hecke algebra $\mathcal{H}(G, \rho)$ of locally constant, compactly supported, $\rho^{\vee}$-spherical functions from $G$ to $\operatorname{End}_{\mathbb{C}}\left(\rho^{\vee}\right)$. (Here, $\rho^{\vee}$ is the contragredient of $\rho$.) There is a correspondence between the set of irreducible smooth representations of $G$ containing $\rho$ and the set of simple modules over $\mathcal{H}(G, \rho)$. (See $[26, \S 3]$ or $[3, \S 4.2 .5]$.) If $\rho$ is chosen carefully, then $\mathcal{H}(G, \rho)$ is isomorphic (or Morita equivalent), to another Hecke algebra $\mathcal{H}\left(M, \rho^{\prime}\right)$, where $M$ has smaller semisimple rank than $G$. In our case, the algebras themselves are very easy to describe after a few general results have been established.

First, if $\rho$ is a character arising from $\S 1.7$, and $\Upsilon$ is the corresponding coset in $\mathfrak{g}^{*}$, then the support of $\mathcal{H}(G, \rho)$ consists of all $g \in G$ such that $\operatorname{Ad}^{*}(g) \Upsilon$ meets $\Upsilon$. This result previously appeared in [14], though in a more specialized context. The mock exponential map's properties are just good enough that the usual proof carries over to the present level of generality; for completeness, the details are included in $\S 1.8$.

Second, under certain hypotheses on $p$, one can compute the support of $\mathcal{H}(G, \rho)$ explicitly, and one can describe the transfer group $M$ as an explicit centralizer. This is carried out in $\S \S 2.2-2.3$.

These results are enough to establish a correspondence between the representations of $G$ that we are considering and certain representations of $M$. All of the former are induced from open, compact mod center subgroups of $G$. In $\S 2.6$, we describe the inducing data more or less explicitly.

We have been careful to include the case where $M$ is not a torus. The representations that then arise from characters of $M$ are what Moy calls "singular" supercuspidals. In $\S 3$, we show that singular supercuspidals exist whenever $G$ is any symplectic or orthogonal group (with a few low-dimensional exceptions), or the exceptional Chevalley group of type $G_{2}$. They also exist for $\mathrm{U}(2,1)$, and probably for all higher-rank unitary groups as well.

It is possible to gather some information about the characters of the supercuspidal representations that we construct here. For example, using methods 
JEFFREY D. ADLER

of Murnaghan [29] and this paper, one can show that, in a large region, such a character is a scalar multiple of the Fourier transform of a certain elliptic orbital integral. We pursue this matter elsewhere [1]. Moreover, one can obtain a vanishing result by refining methods of Kutzko [20].

This paper was originally my doctoral dissertation, written under the supervision of Paul Sally. Allen Moy pointed out to me the existence of singular supercuspidal representations, suggested that I construct some for $\mathrm{G}_{2}$, and provided me with helpful advice at several points. I have also benefited from conversations with Robert Kottwitz and Gopal Prasad, and also Philip Kutzko, Alan Roche (who showed me an early draft of [32]), and Stephen DeBacker.

It is a pleasure to thank all of these people.

\section{Structure theory.}

1.1. Notation. The following is in force everywhere except in $\S 1.2$ and $\S 1.3$. Let $F$ be a non-archimedean local field of residual characteristic $p$, and $\varpi$ a uniformizing element for $F$. Let $\nu$ be a valuation of $F$, normalized so that $\nu(\varpi)=1$. Let $K$ be a maximal unramified extension of $F$. Given any abstract algebraic extension $E$ of $F$, we may (and will) assume that $E$ and $K$ lie in a common algebraic closure. The valuation $\nu$ on $F$ has a unique extension to $E$, which we will also denote by $\nu$. Let $\mathcal{O}_{E}$ denote the ring of integers in $E$, and $\mathcal{O}=\mathcal{O}_{F}$.

Let $\mathbf{G}$ be a connected reductive group defined over $F$, and $G=\mathbf{G}(F)$ its group of $F$-points. Let $\mathfrak{g}=\operatorname{Lie}(\mathbf{G})$, and let $\mathfrak{g}^{*}$ be the dual of $\mathfrak{g}$. For any $E / F$, denote the corresponding group, algebra, and space of $E$-points by $\mathbf{G}(E), \mathfrak{g}(E)$, and $\mathfrak{g}^{*}(E)$, respectively. Let $\mathcal{B}(\mathbf{G}, E)$ be the Bruhat-Tits building of $\mathbf{G}(E)$. We will write $\mathcal{B}$ for $\mathcal{B}(\mathbf{G}, F)$. For any maximal $E$-split $F$-torus $\mathbf{T} \subset \mathbf{G}(E)$, let $\mathcal{A}(\mathbf{T}(E))$ denote the apartment of $\mathbf{T}(E)$ in $\mathcal{B}(\mathbf{G}, E)$. If $x \in \mathcal{A}(\mathbf{T}(E)) \cap \mathcal{B}$, then we will abuse notation and write $x \in \mathcal{A}(\mathbf{T}(F))$.

To any point $x \in \mathcal{B}(\mathbf{G}, K)$, Moy and Prasad [28] associate a parahoric subgroup $\mathbf{G}(K)_{x}$ of $\mathbf{G}(K)$ and a filtration $\mathbf{G}(K)_{x, r}\left(r \in \mathbb{R}_{\geq 0}\right)$. One also has filtrations $\mathfrak{g}(K)_{x, r}$ and $\mathfrak{g}^{*}(K)_{x, r}(r \in \mathbb{R})$ on the Lie algebra and its dual. These groups and lattices are briefly described in $\S 1.4$.

Given any $E$-split torus $\mathbf{S} \subset \mathbf{G}$ (maximal or not) and any $b$ in the root system $\Phi(\mathbf{G}, \mathbf{S})$ of $\mathbf{S}$, let $\mathbf{U}_{b} \subset \mathbf{G}$ denote the corresponding root group, and $\mathfrak{u}_{b} \subset \mathfrak{g}$ the corresponding root space. These are all defined over $E$. Whenever we denote an algebraic $F$-group by a boldface letter, the corresponding lightface letter will generally denote the group of $F$-points. A similar convention will hold for Lie algebras.

For any group $H$, let $\mathcal{D} H$ denote its derived group. Similarly, $\mathcal{D h}$ denotes 
the derived algebra of the Lie algebra $\mathfrak{h}$. Denote the centers of $H$ and $\mathfrak{h}$ by $\mathcal{Z} H$ and $\mathcal{Z} \mathfrak{h}$, respectively. Let $H^{\wedge}$ denote the group of complex characters of $H$. For any group elements $a$ and $b$, let $\operatorname{Int}(a) b=a b a^{-1}$ and $[a, b]=a b a^{-1} b^{-1}$.

Let Ad denote the adjoint and coadjoint actions of $G$ on $\mathfrak{g}$ and $\mathfrak{g}^{*}$, respectively. We will often write $g X$ instead of $\operatorname{Ad}(g) X$.

Fix a nontrivial additive character $\Lambda$ of $F$ with conductor $\varpi \mathcal{O}$. For any $F \subset E \subset K$ and any lattice $\mathcal{L} \subset \mathfrak{g}(E)$, let $\mathcal{L}^{\bullet}=\left\{X \in \mathfrak{g}^{*}(E) \mid X(\mathcal{L}) \subset \varpi \mathcal{O}_{E}\right\}$.

1.2. Review of Chevalley groups. For this section only, let $F$ be an arbitrary field, and $G$ the group of $F$-points of a split group $\mathbf{G}$ defined over $F$. Let $\mathbf{T}$ be a maximal $F$-split torus in $\mathbf{G}$, and let $\mathbf{X}^{*}(\mathbf{T})$ and $\mathbf{X}_{*}(\mathbf{T})$ denote the lattices of rational characters and cocharacters of $\mathbf{T}$, respectively. If $b^{\vee} \in \mathbf{X}_{*}(\mathbf{T})$ and $c \in \mathbf{X}^{*}(\mathbf{T})$, then the composition $c \circ b^{\vee}$ is an $F$-algebraic homomorphism $\mathbf{G L}_{1} \longrightarrow \mathbf{G L}_{1}$, which must have the form $x \longmapsto x^{\left\langle b^{\vee}, c\right\rangle}$, where $\left\langle b^{\vee}, c\right\rangle$ is some integer. We thus have a natural, nondegenerate, $\mathbb{Z}$-bilinear form $\mathbf{X}_{*}(\mathbf{T}) \otimes \mathbf{X}^{*}(\mathbf{T}) \longrightarrow \mathbb{Z}$. Let $\Phi=\Phi(\mathbf{G}, \mathbf{T}) \subset \mathbf{X}^{*}(\mathbf{T})$ be the root system of $\mathbf{T}$, and let $\Delta$ be a system of simple roots in $\Phi$. Let $T=\mathbf{T}(F)$, and let Add denote the algebraic group whose group of $F$-points is just the additive group of $F$.

The following are summaries or easy corollaries of results contained in [6].

Proposition 1.2.1. For each $b \in \Phi, \mathfrak{g}$ contains elements $H_{b}$ and $E_{b}$ such that

$$
\left\{H_{b} \mid b \in \Delta\right\} \cup\left\{E_{b} \mid b \in \Phi\right\}
$$

is a basis for $\mathfrak{g}$ if $G$ is semisimple; and for all roots $b$ and $c$,

$$
\begin{aligned}
& {\left[H_{b}, H_{c}\right]=0} \\
& {\left[H_{b}, E_{c}\right]=\left\langle b^{\vee}, c\right\rangle E_{c}} \\
& {\left[E_{b}, E_{c}\right]= \begin{cases}N_{b, c} E_{b+c} & \text { if } b+c \in \Phi, \\
H_{b} & \text { if } c=-b, \\
0 & \text { otherwise, }\end{cases} }
\end{aligned}
$$

where each $N_{b, c}$ is an integer.

Call such a set a Chevalley basis. There can be many choices for the $E_{b}$, leading to different values of the constants $N_{b, c}$. Let us fix a choice. Note that for all $H \in \operatorname{Lie}(\mathbf{T}),\left[H, E_{c}\right]=d c(H) \cdot E_{c}$, where $d c$ is the derivative of $c$.

Proposition 1.2.2. For each $b \in \Phi$, there exists a unique map $e_{b}:$ Add $\longrightarrow$ $\mathbf{G}$ such that $d_{b}(1)=E_{b}$. Define a map $b^{\vee}: \mathbf{G L}_{1} \longrightarrow \mathbf{G}$, by $b^{\vee}(\lambda)=$ $w_{b}(\lambda) w_{b}(1)^{-1}$, where $w_{b}(\lambda)=e_{b}(\lambda) e_{-b}\left(-\lambda^{-1}\right) e_{b}(\lambda)$. Then $b^{\vee}$ is the coroot of $\mathbf{T}$ corresponding to $b$, and $d b^{\vee}(1)=H_{b}$. 
When $b \in \mathbf{X}^{*}(\mathbf{T}) \backslash \Phi$, let $e_{b}$ denote the trivial map Add $\longrightarrow \mathbf{G}$. When $b+c$ is not a root, let $N_{b, c}=0$. For all $b, c \in \Phi$ and all positive $i \in \mathbb{Z}$, let

$$
M_{b, c ; i}=\frac{1}{i !} \cdot N_{b, c} N_{b, b+c} \cdots N_{b,(i-1) b+c}
$$

and let $M_{b, c ; 0}=1$.

Proposition 1.2.3. Let $b, c \in \Phi$ be linearly independent. Then

$$
\left[e_{b}(\lambda), e_{c}(\mu)\right]=\prod_{i, j>0} e_{i b+j c}\left(C_{i, j ; b, c} \lambda^{i} \mu^{j}\right)
$$

where the product is taken in increasing order of the roots, the $C_{i, j ; b, c}$ are integers, and

$$
\begin{aligned}
C_{i, 1 ; b, c} & =M_{b, c ; i} \\
C_{1, j ; b, c} & =(-1)^{j} M_{c, b ; j} .
\end{aligned}
$$

In particular, $C_{1,1 ; b, c}=N_{b, c}$.

Proposition 1.2.4. For any $b \in \Phi$ and any $t \in T$,

$$
\left[t, e_{b}(\mu)\right]=e_{b}(b(t) \mu-\mu)
$$

Proposition 1.2.5. The adjoint representation is determined by the following formulas:

$$
\begin{aligned}
\operatorname{Ad}\left(e_{b}(\lambda)\right) E_{c} & = \begin{cases}E_{b} & \text { if } c=b, \\
E_{-b}+\lambda H_{b}-\lambda^{2} E_{b} & \text { if } c=-b, \\
\sum_{i \geq 0} M_{b, c ; i} \lambda^{i} E_{i b+c} & \text { if } c \neq \pm b\end{cases} \\
\operatorname{Ad}(t) E_{c} & =c(t) E_{c} \\
\operatorname{Ad}\left(e_{b}(\lambda)\right) H & =H-d b(H) \lambda E_{b} \\
\operatorname{Ad}(t) H & =H
\end{aligned}
$$

for all $H \in \operatorname{Lie}(T)$ and all $t \in T$.

1.3. Some homeomorphisms between filtered groups. Let $A=A_{1}$ and $B=B_{1}$ be groups, and let $\left\{A_{i}\right\}_{i \in \mathbb{N}}$ and $\left\{B_{i}\right\}_{i \in \mathbb{N}}$ be filtrations by normal subgroups, such that $\bigcap_{i \in \mathbb{N}} A_{i}$ and $\bigcap_{i \in \mathbb{N}} B_{i}$ are trivial. Give each $A / A_{i}$ and $B / B_{i}$ the discrete topology, and give $A$ and $B$ the inverse limit topology. 
Let $f: \mathbb{N} \longrightarrow \mathbb{N}$ be an increasing function such that $f(i)>i$ for all $i \in \mathbb{N}$. Suppose we have a collection of isomorphisms

$$
\varphi_{i, j}: A_{i} / A_{j} \stackrel{\sim}{\longrightarrow} B_{i} / B_{j}
$$

for all $1 \leq i \leq j \leq f(i)$; moreover, suppose that these maps are compatible with each other, in the sense that for all $1 \leq i \leq i^{\prime} \leq j^{\prime} \leq j \leq f(i)$,

$$
\varphi_{i, j}\left(a A_{j}\right) \equiv \varphi_{i^{\prime}, j^{\prime}}\left(a A_{j^{\prime}}\right) \bmod B_{j^{\prime}}
$$

for all $a \in A_{i^{\prime}}$.

For each quotient $A_{i} / A_{i+1}$, choose a set $X_{i}$ of coset representatives. We will always let the identity element represent the identity coset. For each $a \in X_{i}$, choose an element $\varphi(a)$ in the coset $\varphi_{i, f(i)}\left(a A_{f(i)}\right)$. (When $a$ is the identity, choose $\varphi(a)$ to be the identity in $B$.) Any element of $A_{r}$ can be written uniquely in the form $a=\prod_{i=r}^{\infty} a_{i}$, where $a_{i} \in X_{i}$. Similarly for elements of $B_{r}$.

Using these choices, define a function $\varphi: A \longrightarrow B$ by

$$
\varphi\left(\prod_{i \in \mathbb{N}} a_{i}\right)=\prod_{i \in \mathbb{N}} \varphi\left(a_{i}\right) .
$$

Proposition 1.3.1. The function $\varphi: A \longrightarrow B$ is a homeomorphism compatible with the collection $\left\{\varphi_{i, j}\right\}$.

Proof. By construction, $\varphi$ is compatible with each $\varphi_{i, j}$. In particular, it induces bijections on all successive quotients, and is therefore bijective. The continuity of $\varphi$ and its inverse is clear from the choice of topologies.

Proposition 1.3.2. Let $x \in A_{r}, y \in A_{s}$. Then

$$
\varphi(x y) \equiv \varphi(x) \varphi(y) \bmod B_{f(\max (r, s))}\left[B_{r}, B_{s}\right] .
$$

Proof. Write $x=\prod x_{i}$ and $y=\prod y_{i}$, where $x_{i}, y_{i} \in X_{i}$. Let $t=\max (r, s)$, and write

$$
x^{\prime}=\prod_{r \leq i<t} x_{i}, \quad y^{\prime}=\prod_{s \leq i<t} y_{i}
$$

Note that $x^{\prime}$ or $y^{\prime}$ is trivial. Then

$$
\begin{aligned}
\varphi(x) \varphi(y) & =\prod \varphi\left(x_{i}\right) \prod \varphi\left(y_{i}\right) \\
& \equiv \varphi\left(x^{\prime} y^{\prime}\right) \prod_{i \geq t} \varphi\left(x_{i}\right) \varphi\left(y_{i}\right) \bmod \left[B_{r}, B_{s}\right] \\
& \equiv \varphi\left(x^{\prime} y^{\prime}\right) \prod_{i \geq t} \varphi\left(x_{i} y_{i}\right) \bmod B_{f(t)} \\
& \equiv \varphi(x y) \bmod B_{f(t)} .
\end{aligned}
$$


This last step follows from the fact that $\varphi\left(a^{\prime} a\right)=\varphi\left(a^{\prime}\right) \varphi(a)$ whenever $a^{\prime}$ has the form $\prod_{i<t} a_{i}$, with $a_{i} \in X_{i}$, and $a \in A_{t}$.

Remark 1.3.3. Not all $\left\{\varphi_{i, j}\right\}$-compatible homeomorphisms from $A$ to $B$ arise in this way. For example, suppose that

$$
A=A^{(1)} \cdots A^{(n)} \quad \text { and } \quad B=B^{(1)} \cdots B^{(n)},
$$

where the factors have only trivial pairwise intersection. Each $A^{(k)}$ (resp. $\left.B^{(k)}\right)$ inherits a filtration from $A$ (resp. $B$ ). Suppose that by restricting the $\varphi_{i, j}$ we obtain isomorphisms

$$
\varphi_{i, j}^{(k)}: A_{i}^{(k)} / A_{j}^{(k)} \stackrel{\sim}{\longrightarrow} B_{i}^{(k)} / B_{j}^{(k)}
$$

for all $1 \leq i \leq j \leq f(i)$ and all $1 \leq k \leq n$, and that for any $k$ the maps $\varphi_{i, j}^{(k)}$ are compatible with each other in the same sense as above. We can form homeomorphisms $\varphi^{(k)}: A^{(k)} \longrightarrow B^{(k)}$ as above. Since $A$ is the topological direct product of the $A^{(k)}$, and similarly for $B$, we can form the corresponding homeomorphism $\varphi: A \longrightarrow B$. This map depends on the ordering of the factors in $A$ and $B$. For any ordering, we get a map that is compatible with all $\varphi_{i, j}$, and (1.3.2) still applies.

We will use this construction in $\S 1.5$.

1.4. Some properties of the Moy-Prasad filtrations. We start by giving a description of the filtrations similar to that given in [28]. Recall that $K$ is a maximal unramified extension of $F$, and let $L / K$ be the splitting field of $\mathbf{G}$. Let $\mathbf{S}$ be a maximal $K$-split $F$-torus that contains a maximal $F$-split torus. Let $\mathbf{T}$ be the centralizer of $\mathbf{S}$ in $\mathbf{G}$. Then, since $\mathbf{G}(K)$ is quasi-split, $\mathbf{T}$ is a maximal $F$-torus that splits over $L$. Let $\mathfrak{t} \subset \mathfrak{g}$ be the Lie algebra of T.

Let $\mathbf{N}$ be the normalizer of $\mathbf{T}$. Then $\mathbf{N}(K)$ is also the normalizer of $\mathbf{S}(K)$ in $\mathbf{G}(K)$. Let $V_{L}=\mathbf{X}_{*}(\mathbf{T}) \otimes_{\mathbb{Z}} \mathbb{R}$, and let $V_{K}$ be the space of $\operatorname{Gal}(L / K)$-fixed points in $V_{L}$. Let $A_{L}=\mathcal{A}(\mathbf{T}(L))$. Then $A_{K}$, the space of Galois-fixed points in $A_{L}$, is the apartment of $\mathbf{S}(K)$. These are affine spaces under $V_{L}$ and $V_{K}$, respectively.

The parahoric subgroup of $\mathbf{G}(K)$ associated to $x \in \mathcal{B}(\mathbf{G}, K)$ is denoted $\mathbf{G}(K)_{x}$, and it can be characterized as the "mod $\varpi$ connected component" of the stabilizer of $x$ under the action of $\mathbf{G}(K)$ on $\mathcal{B}(\mathbf{G}, K)$. Let $\mathbf{T}(K)_{0}=$ $\mathbf{T}(K) \cap \mathbf{G}(K)_{x}$, where $x$ is any element of $\mathcal{A}(\mathbf{S}(K))$. For each $m>0$, we have a compact open filtration subgroup

$$
\mathbf{T}(K)_{m}=\left\{t \in \mathbf{T}(K) \mid \nu(\chi(t)-1) \geq m \text { for all } \chi \in \mathbf{X}^{*}(\mathbf{T})\right\}
$$


of $\mathbf{T}(K)$. If $x \in \mathcal{B}$, then these are all defined over $F$, and we let $T_{m}$ denote $T \cap \mathbf{T}(K)_{m}$ for $m \geq 0$. The derivative of a character of $\mathbf{T}$ is a weight of $\mathfrak{t}$. For each $m \in \mathbb{R}$, we define a filtration sublattice $\mathfrak{t}(K)_{m}$ of $\mathfrak{t}(K)$ by

$$
\mathfrak{t}(K)_{m}=\left\{H \in \mathfrak{t}(K) \mid \nu(d \chi(H)) \geq m \text { for all } \chi \in \mathbf{X}^{*}(\mathbf{T})\right\} .
$$

We have a map $\kappa: \mathbf{T}(K) \longrightarrow V_{K}$ given by

$$
\langle\chi, \kappa(t)\rangle=-\nu(\chi(t))
$$

for all $\chi \in \mathbf{X}_{K}^{*}(\mathbf{T})$. This map extends to a map from $\mathbf{N}(K)$ to the group of affine transformations of $A_{K}$.

Similarly, one obtains a map $\kappa_{L}$ from $\mathbf{N}(L)$ to the affine transformations of $A_{L}$. The maps $\kappa$ and $\kappa_{L}$ are compatible in the sense that for $n \in \mathbf{N}(K)$, $\kappa_{L}(n)$ preserves $A_{K}$ in $A_{L}$, and its restriction to $A_{K}$ is just $\kappa(n)$.

Let $b$ be a root in $\Phi(\mathbf{G}, \mathbf{S})$, and let $U_{b}=\mathbf{U}_{b}(K)$. Given $u \in U_{b}$, the set $U_{-b} u U_{-b} \cap \mathbf{N}(K)$ consists of one element, denoted $m(u)$. Let $\alpha(b, u)$ be the affine function on $A_{K}$ whose gradient is $b$ and that vanishes on the hyperplane fixed by the reflection $\kappa(m(u))$. For any affine function $\psi$ on $A_{K}$ of gradient $b$, define

$$
U_{\psi}=\left\{u \in U_{b} \mid u=1 \text { or } \alpha(b, u) \geq \psi\right\} .
$$

One can also define a lattice $\mathfrak{u}_{\psi}$ in the root space $\mathfrak{u}_{b}$. See $[\mathbf{2 7}, \S 3.2]$ for details.

For $r \geq 0$ and $x \in A_{K}$, define $\mathbf{G}(K)_{x, r}$ to be the group generated by $\mathbf{T}(K)_{r}$ and $\left\{U_{\psi} \mid \psi(x) \geq r\right\}$. Then $\mathbf{G}(K)_{x, 0}=\mathbf{G}(K)_{x}$. For $r \in \mathbb{R}$, let $\mathfrak{g}(K)_{x, r}$ be the lattice in $\mathfrak{g}(K)$ spanned by $\mathfrak{t}(K)_{r}$ and $\left\{\mathfrak{u}_{\psi} \mid \psi(x) \geq r\right\}$. Let $\mathbf{G}(K)_{x, r^{+}}=\bigcup_{s>r} \mathbf{G}(K)_{x, s}$ and $\mathfrak{g}(K)_{x, r^{+}}=\bigcup_{s>r} \mathfrak{g}(K)_{x, s}$.

For a general point $x \in \mathcal{B}(\mathbf{G}, K)$, there exists $g \in \mathbf{G}(K)$ such that $g x \in$ $A_{K}$. Define $\mathbf{G}(K)_{x, r}=\operatorname{Int}\left(g^{-1}\right) \mathbf{G}(K)_{g x, r}$ and $\mathfrak{g}(K)_{x, r}=\operatorname{Ad}\left(g^{-1}\right) \mathfrak{g}(K)_{g x, r}$. These definitions do not depend on the choice of $g$ or the initial choice of $\mathbf{S}$.

Let $\mathfrak{g}^{*}(K)_{x, r}=\mathfrak{g}(K)_{x,(-r)^{+}}^{\bullet}$, and $\mathfrak{g}^{*}(K)_{x, r^{+}}=\bigcup_{s>r} \mathfrak{g}^{*}(K)_{x, s}$.

If $x \in \mathcal{B}(\mathbf{G}, F)$, then the groups and lattices above are all defined over $F$, and their $F$-point sets, which are just their fixed point sets under $\operatorname{Gal}(K / F)$, are denoted $G_{x, r}, \mathfrak{g}_{x, r}$, etc.

Proposition 1.4.1. Let $F^{\sharp} / F$ be a finite extension, and let $\mathbf{G}^{\sharp}=\mathbf{G} \times{ }_{F} F^{\sharp}$ be $\mathbf{G}$ regarded as an $F^{\sharp}$-group. Define $G^{\sharp}, \mathfrak{g}^{\sharp}$, etc., accordingly. Let e be the ramification degree of $F^{\sharp}$ over $F$. Then for all $r \in \mathbb{R}$ and for all $x \in \mathcal{B}$,

$$
\begin{aligned}
G_{x, r}^{\sharp} \cap G_{x} & =G_{x, r / e} \quad(r \geq 0), \\
\mathfrak{g}_{x, r}^{\sharp} \cap \mathfrak{g} & =\mathfrak{g}_{x, r / e}, \\
\mathfrak{g}_{x, r}^{\sharp} \cap \mathfrak{g}^{*} & =\mathfrak{g}_{x, r / e}^{*} .
\end{aligned}
$$


Proof. Let $K^{\sharp}$ be the compositum $F^{\sharp} K$. This is a maximal unramified extension of $F^{\sharp}$. Since the filtration subgroups and sublattices were originally defined via Galois descent from $K$ and $K^{\sharp}$, it will be sufficient to prove the result with $F$ and $F^{\sharp}$ replaced by $K$ and $K^{\sharp}$, respectively. Clearly, it is also enough to consider the case where $K^{\sharp}=L$.

We will just prove the statement about the filtrations on the group; the proofs for the statements about the Lie algebra and its dual will be similar, but easier.

The filtration on $T$ is clearly compatible with Galois descent in the desired sense:

$$
T_{r}^{\sharp} \cap T=T_{r / e}, \quad(r>0) .
$$

Thus, it remains only to show that a similar fact is true for the affine root groups. Let $b \in \Phi(\mathbf{G}, \mathbf{S})$ be a $K$-root. Then $b$ corresponds to a $\operatorname{Gal}(L / K)$ orbit $\left\{b_{i}\right\}$ of $L$-roots in $\Phi(\mathbf{G}, \mathbf{T})$. Suppose first that $b$ is not multipliable. Then the root groups $U_{b_{i}}=\mathbf{U}_{b_{i}}(L)$ all commute with each other. Let $u \in U_{b}$. Then $u=\prod u_{i}$, where $u_{i}$ lies in $U_{b_{i}}$ and the $u_{i}$ are all Galois conjugates of each other. Since the $u_{i}$ commute with each other and with the $U_{-b_{j}}(j \neq i)$,

$$
\begin{aligned}
U_{-b} u U_{-b} \cap \mathbf{N}(K) & =\left(\prod U_{-b_{i}} u_{i} U_{-b_{i}}\right)^{\operatorname{Gal}(L / K)} \cap \mathbf{N}(K) \\
& =\prod\left(U_{-b_{i}} u_{i} U_{-b_{i}} \cap \mathbf{N}(L)\right) .
\end{aligned}
$$

In other words, $m(u)=\prod m\left(u_{i}\right)$. Let $H_{i} \subset A_{L}$ be the hyperplane fixed by $\kappa\left(m\left(u_{i}\right)\right)$, and let $H \subset A_{K}$ be the hyperplane fixed by $\kappa(m(u))$. It is clear that for each $i, H_{i} \cap A_{K}=H$.

Let $\psi$ be an affine function of gradient $b$ on $A_{K}$. Then $\psi$ corresponds to a Galois orbit $\left\{\psi_{i}\right\}$ of affine functions of respective gradients $b_{i}$ on $A_{L}$, all of which agree with $\psi$ on $A_{K}$. Thus, $\left.\alpha\left(b_{i}, u_{i}\right)\right|_{A_{K}}=\alpha(b, u)$ for all $i$. This implies that $u \in U_{\psi}$ if and only if each $u_{i} \in U_{\psi_{i}}$, which concludes our proof for the case where $b$ is not multipliable.

Now suppose that $b$ is multipliable. Without loss of generality, we may replace $\mathbf{G}$ by the group generated by $\mathbf{U}_{b}$ and $\mathbf{U}_{-b}$. From an examination of the absolute Dynkin diagram of $\mathbf{G}$, there exists an extension $K_{b} / K$ in $L$ such that $\mathbf{G}(K)$ is isogenous to $\mathbf{S U}(3)_{L / K_{b}}$. An explicit $\mathbf{S U}(3)$ computation completes the proof.

Proposition 1.4.2. For all $x \in \mathcal{B}$ and all $r, s \in \mathbb{R}$,

$$
\begin{aligned}
{\left[G_{x, r}, G_{x, s}\right] } & \subseteq G_{x, r+s} \quad(r, s \geq 0) \\
{\left[\mathfrak{g}_{x, r}, \mathfrak{g}_{x, s}\right] } & \subseteq \mathfrak{g}_{x, r+s} .
\end{aligned}
$$


Proof. This follows from 2.4, 2.7, and 2.20 in [31].

Proposition 1.4.3. For any positive $r$ and any $s \in \mathbb{R}$, the adjoint action of $G$ induces the trivial action of $G_{x, r}$ on $\mathfrak{g}_{x, s} / \mathfrak{g}_{x, r+s}$.

Proof. From (1.4.1), we may assume that $\mathbf{G}$ is $F$-split. Let $\mathbf{T}$ be a maximal $F$-split torus such that $x \in \mathcal{A}(T)$. The group $G_{x, r}$ is generated by $\left\{U_{\psi} \mid \psi(x) \geq r\right\}$ and $T_{r}$, so it is enough to see that each of these groups acts trivially on $\mathfrak{g}_{x, s} / \mathfrak{g}_{x, r+s}$. This follows from (1.2.5).

1.5. A mock exponential map. Assume for the moment that $\mathbf{G}$ is $F$-split, and let $\mathbf{T} \subset \mathbf{G}$ be a maximal $F$-split torus. Let $T=\mathbf{T}(F)$ and $\mathfrak{t}=\operatorname{Lie}(T)$. Choose an integral basis $\left\{\chi_{i}\right\}$ for the lattice $\mathbf{X}^{*}(\mathbf{T})$. Define a function

$$
\varphi_{T}: \mathfrak{t}_{1} \longrightarrow T_{1}
$$

by

$$
\chi_{i}\left(\varphi_{T}(H)\right)=1+d \chi_{i}(H) \quad \text { for all } i .
$$

Clearly, $\varphi_{T}\left(\mathfrak{t}_{r}\right)=T_{r}$, so $\varphi_{T}$ induces functions $\varphi_{T ; r, s}: \mathfrak{t}_{r} / \mathfrak{t}_{s} \longrightarrow T_{r} / T_{s}$ for all $0<r \leq s$. Moreover, $\varphi_{T ; r, s}$ is an isomorphism if $s \leq 2 r$.

Lemma 1.5.1 If $0<r \leq s \leq 2 r$, then $\varphi_{T ; r, s}$ does not depend on the choice of basis for $\mathbf{X}^{*}(\mathbf{T})$.

Proof. Define a map $\varphi_{T}^{\prime}$ with respect to some other basis; choose an element $\chi^{\prime}$ in this new basis. We can write $\chi^{\prime}=\prod \chi_{i}^{a_{i}}, a_{i} \in \mathbb{Z}$. Let $H \in \mathfrak{t}_{r}$. Then

$$
\begin{aligned}
\chi^{\prime}\left(\varphi_{T}(H)\right)=\prod\left(\chi_{i}\left(\varphi_{T}(H)\right)\right)^{a_{i}} & =\prod\left(1+d \chi_{i}(H)\right)^{a_{i}} \\
& \equiv 1+\sum a_{i} d \chi_{i}(H) \bmod \left(\varpi^{2 r}\right) \\
& =1+d \chi^{\prime}(H) \\
& =\chi^{\prime}\left(\varphi_{T}^{\prime}(H)\right) .
\end{aligned}
$$

Since the same is true for all $\chi^{\prime} \in \mathbf{X}^{*}(\mathbf{T}), \varphi_{T}(H) \equiv \varphi_{T}^{\prime}(H) \bmod T_{2 r}$.

We will now extend $\varphi_{T}$ to a larger domain in $\mathfrak{g}$.

Let $\Phi$ be the root system of $\mathbf{G}$ with respect to $\mathbf{T}$. Choose a Chevalley basis for $\mathfrak{g}$ with respect to $T$, and denote it as in (1.2.1). For each $b \in \Phi$, there is a map $\exp _{b}: \mathfrak{u}_{b} \longrightarrow U_{b}$, defined by $\alpha E_{b} \longmapsto e_{b}(\alpha)$, which has the property that

$$
\operatorname{Int}(t) \exp _{b}(X)=\exp _{b}(b(t) X)
$$


for all $t \in T$. This map is compatible with the filtrations on $\mathfrak{u}_{b}$ and $U_{b}$.

Choosing an ordering of the roots, we can paste all of these maps together to get a map

$$
\varphi_{T}:\left(\mathfrak{t}_{1} \oplus \bigoplus_{b \in \Phi} \mathfrak{u}_{b}\right) \longrightarrow G
$$

defined by

$$
H+\sum_{b \in \Phi} X_{b} \longmapsto \varphi_{T}(H) \prod_{b \in \Phi} \exp _{b} X_{b},
$$

where the order of the product is the order we have just chosen on $\Phi$. Note that, while this map is not defined on all of $\mathfrak{g}$, it is defined on $\mathfrak{g}_{x, 0^{+}}$for every $x$ in $\mathcal{A}(T)$.

Concretely, we see that $\varphi_{T}\left(\alpha E_{b}\right)=e_{b}(\alpha)$, and if $\nu(\alpha) \geq r>0$, then $\varphi_{T}\left(\alpha H_{b}\right) \equiv b^{\vee}(1+\alpha) \bmod G_{x, 2 r}$.

For $0<r \leq s \leq 2 r$, the induced maps $\varphi_{T, x ; r, s}: \mathfrak{g}_{x, r} / \mathfrak{g}_{x, s} \longrightarrow G_{x, r} / G_{x, s}$ are isomorphisms which are independent of the ordering on $\Phi$ and the basis for $\mathbf{X}^{*}(\mathbf{T})$. (In fact, we need only assume that $0<r \leq s \leq\left(2 \cdot r_{x}^{\uparrow}\right)_{x}^{\uparrow}$, where for any $t \in \mathbb{R}, t_{x}^{\uparrow}=\max \left\{u \mid \mathfrak{g}_{x, t}=\mathfrak{g}_{x, u}\right\}$. In the sequel, the assumption $0<r \leq s \leq 2 r$ may be similarly weakened wherever it occurs.) It will turn out (1.6.6) that $\varphi_{T, x ; r, s}$ is independent of the choice of torus $\mathbf{T}$ such that $x \in \mathcal{A}(T)$.

More generally, for any nonempty subset $S \subset \mathcal{A}(T)$, and any positivevalued function $f: S \longrightarrow \mathbb{R}$, let

$$
G_{S, f}=\bigcap_{x \in S} G_{x, f(x)}, \quad \mathfrak{g}_{S, f}=\bigcap_{x \in S} \mathfrak{g}_{x, f(x)} .
$$

If $f^{\prime}$ is another such function, and $0<f \leq f^{\prime} \leq 2 f$, then we have an isomorphism

$$
\varphi_{T, S ; f, f^{\prime}}: \mathfrak{g}_{S, f} / \mathfrak{g}_{S, f^{\prime}} \longrightarrow G_{S, f} / G_{S, f^{\prime}}
$$

Now drop the assumption that $\mathbf{G}$ is $F$-split, let $\mathbf{T} \subset \mathbf{G}$ be a maximal $F$-torus, and let $F^{\sharp} / F$ be some finite Galois extension over which $\mathbf{T}$ splits. Let $e$ be the ramification degree of $F^{\sharp} / F$. Then, as above, we can define a map $\varphi_{T}^{\sharp}$ from a subset of $\mathfrak{g}^{\sharp}=\mathfrak{g}\left(F^{\sharp}\right)$ into $G^{\sharp}=\mathbf{G}\left(F^{\sharp}\right)$. For every nonempty subset $S \subset \mathcal{B} \cap \mathcal{A}\left(\mathbf{T}\left(F^{\sharp}\right)\right)$ and every positive-valued function $f: S \longrightarrow \mathbb{R}$, the group $G_{S, \text { ef }}^{\sharp}$ and lattice $\mathfrak{g}_{S, \text { ef }}^{\sharp}$ are $\operatorname{Gal}\left(F^{\sharp} / F\right)$-invariant. If $f^{\prime}$ is another such function, and $0<f \leq f^{\prime} \leq 2 f$, then the induced maps $\varphi_{T, S ; e f, e f^{\prime}}^{\sharp}: \mathfrak{g}_{S, e f}^{\sharp} / \mathfrak{g}_{S, e f^{\prime}}^{\sharp} \longrightarrow G_{S, e f}^{\sharp} / G_{S, e f^{\prime}}^{\sharp}$ are invariant under $\operatorname{Gal}\left(F^{\sharp} / F\right)$. It follows from (1.4.1) that we get a map $\varphi_{T, S ; f, f^{\prime}}: \mathfrak{g}_{S, f} / \mathfrak{g}_{S, f^{\prime}} \longrightarrow G_{S, f} / G_{S, f^{\prime}}$ which is independent of our choice of splitting field $F^{\sharp}$. 
For any $x \in \mathcal{A}\left(\mathbf{T}\left(F^{\sharp}\right)\right) \cap \mathcal{B}$, one can use the construction of $\S 1.3$ to define, in a non-canonical way, a homeomorphism

$$
\varphi_{T, x}: \mathfrak{g}_{x, 0^{+}} \longrightarrow G_{x, 0^{+}}
$$

that is compatible with every $\varphi_{T, x ; r, s}$. Since, as remarked before, the maps $\varphi_{T, x ; r, s}$ will turn out to be independent of $\mathbf{T}, \varphi_{T, x}$ will also be independent of $\mathbf{T}$, though it will still depend on the choices made in $\S 1.3$.

\subsection{Properties of the mock exponential map.}

Remark 1.6.1. From the results of $\S 1.4$, several of the following properties of $\varphi_{T, x}$ will follow from the special case where $\mathbf{T}$ is $F$-split. Moreover, their validity will not depend on any of the choices that go into the definition of $\varphi_{T, x}$ from $\varphi_{T}$. Therefore, in proofs we will often be able to assume that $\mathbf{T}$ is $F$-split and that $\varphi_{T, x}$ is just the restriction of $\varphi_{T}$ to $\mathfrak{g}_{x, 0^{+}}$.

Proposition 1.6.2. Let $\mathbf{T} \subset \mathbf{G}$ be a maximal $F$-torus, and write $\varphi=$ $\varphi_{T, x}$, where $x \in \mathcal{A}(T)$. Suppose $Y \in \mathfrak{g}_{x, r}$ and $Z \in \mathfrak{g}_{x, s}$, with $r, s>0$. Then

$$
\begin{aligned}
\varphi(Y) \varphi(Z) & \equiv \varphi(Y+Z) \bmod G_{x, r+s} \\
{[\varphi(Y), \varphi(Z)] } & \equiv \varphi[Y, Z] \bmod G_{x, r+s+\min (r, s)} .
\end{aligned}
$$

Proof. The first statement follows immediately from (1.3.2).

To prove the second statement, make the assumptions in (1.6.1). Choose an ordering on $\Phi(\mathbf{G}, \mathbf{T})$. Choose a Chevalley basis corresponding to $T$, and adopt the notation of (1.2.1). The result is clearly true if $Y$ and $Z$ both lie in $\mathfrak{t}=\operatorname{Lie}(T)$, or both lie in some $\mathfrak{u}_{b}$. If $Y \in \mathfrak{u}_{b}$ and $Z \in \mathfrak{u}_{-b}$ for some $b \in \Phi$, then the result follows from an $\mathbf{S L}(2)$ calculation.

Now suppose that $Y=\lambda E_{b} \in \mathfrak{u}_{\psi}, Z=\mu E_{c} \in \mathfrak{u}_{\eta}$, where $\lambda, \mu \in F$, $\psi(x) \geq r, \eta(x) \geq s$, and $b \neq \pm c$. Then $[Y, Z]=N_{b, c} \lambda \mu E_{b+c}$, while

$$
\begin{aligned}
{[\varphi(Y), \varphi(Z)] } & =\prod_{i, j>0} e_{i b+j c}\left(C_{i, j ; b, c} \lambda^{i} \mu^{j}\right) \\
& =e_{b+c}\left(N_{b, c} \lambda \mu\right) \cdot \text { higher-order terms }
\end{aligned}
$$

where the order of the product corresponds to the order chosen on $\Phi(\mathbf{G}, \mathbf{T})$, and the higher-order terms are

$$
\prod_{\substack{i, j>0 \\ i>1 \text { or } j>1}} e_{i b+j c}\left(C_{i, j ; b, c} \lambda^{i} \mu^{j}\right) \in U_{2 \psi+\eta} \cdot U_{\psi+2 \eta} \subset G_{x, r+s+\min (r, s)} .
$$


Suppose that $Y \in \mathfrak{t}_{r}$ and $Z=\mu E_{c}$. Then

$$
\begin{aligned}
{[\varphi(Y), \varphi(Z)] } & =\left[\varphi(Y), e_{c}(\mu)\right] \\
& =e_{c}(c(\varphi(Y)) \mu-\mu) \quad \text { by }(1.2 .4) \\
& \equiv e_{c}((1+d c(Y)) \mu-\mu) \bmod G_{x, 2 r+s} \\
& =e_{c}(\mu d c(Y)) \\
& =\varphi(d c(Y) Z) \\
& =\varphi([Y, Z]) .
\end{aligned}
$$

This completes the proof for the case where $Y$ and $Z$ are elements of $\mathfrak{t}$ or multiples of Chevalley basis elements. The general case now follows from the fact that if $Y_{1}, \ldots, Y_{n} \in \mathfrak{g}_{x, r}$ then

$$
\varphi\left(Y_{1}+\cdots+Y_{n}\right) \equiv \varphi\left(Y_{1}\right) \varphi\left(Y_{2}\right) \cdots \varphi\left(Y_{n}\right) \bmod G_{x, 2 r}
$$

and

$$
\left[G_{x, 2 r}, G_{x, 2 s}\right] \subset G_{x, 2 r+2 s} \subset G_{x, r+s+\min (r, s)} .
$$

Proposition 1.6.3. Let $\mathbf{T} \subset \mathbf{G}$ be a maximal $F$-torus, and write $\varphi=$ $\varphi_{T, x}$, where $x \in \mathcal{A}(T)$. Suppose $r>0, Y \in \mathfrak{g}_{x, r}$, and $Z \in \mathfrak{g}_{x, s}$. Then

$$
\operatorname{Ad}(\varphi(Y)) Z \equiv Z+\operatorname{ad}(Y) Z \bmod \mathfrak{g}_{x, 2 r+s} .
$$

Proof. Make the assumptions in (1.6.1). Pick a Chevalley basis, as in the proof of (1.6.2). We begin by proving the proposition in the special case where $Y$ is an element of $\mathfrak{t}=\operatorname{Lie}(T)$ or a scalar multiple of a basis element, where we can exploit (1.2.5).

Suppose $Y \in \mathfrak{t}_{r}$. Then the proposition certainly holds for $Z \in \mathfrak{t}$. If $Z=\mu E_{c}$ with $\mu \in F$, then

$$
\begin{aligned}
\operatorname{Ad}(\varphi(Y)) Z & =c(\varphi(Y)) \mu E_{c} \quad \text { by }(1.2 .5) \\
& \equiv(1+d c(Y)) \mu E_{c} \bmod G_{x, 2 r+s} \\
& =Z+d c(Y) Z \\
& =Z+\operatorname{ad}(Y) Z .
\end{aligned}
$$

Now let $Y=\lambda E_{b}$. If $Z \in \mathfrak{t}_{s}$, then

$$
\operatorname{Ad}(\varphi(Y)) Z=\operatorname{Ad}\left(e_{c}(\lambda)\right) Z=Z-d c(Z) Y=Z+\operatorname{ad}(Y) Z .
$$

If $Z$ is a multiple of $E_{b}$, then $[Y, Z]=0$ and $\operatorname{Ad}(\varphi(Y)) Z=Z$. If $Z \in \mathfrak{u}_{-b}$, then the result is an $\mathbf{S L}(2)$ calculation. If $Z=\mu E_{c}$, with $c \neq \pm b$, then

$$
[Y, Z]=\lambda \mu N_{b, c} E_{b+c}
$$


and

$$
\operatorname{Ad}(\varphi(Y)) Z=\sum_{i \geq 0} M_{b, c ; i} \lambda^{i} \mu E_{i b+c}
$$

where, in particular, $M_{b, c ; 0}=1, M_{b, c ; 1}=N_{b, c}$, and $M_{b, c ; i} \lambda^{i} \mu E_{i b+c} \in \mathfrak{g}_{x, 2 r+s}$ for all $i>1$.

This completes the proof for the case where $Y$ is an element of $\mathfrak{t}$ or a multiple of a Chevalley basis element. Now let $Y_{1}, Y_{2} \in \mathfrak{g}_{x, r}$, and suppose that the proposition is true for $Y_{1}$ and $Y_{2}$. Then

$$
\begin{aligned}
\operatorname{Ad} \varphi\left(Y_{1}+Y_{2}\right) Z \equiv & \operatorname{Ad} \varphi\left(Y_{1}\right)\left(\operatorname{Ad} \varphi\left(Y_{2}\right) Z\right) \bmod \mathfrak{g}_{x, 2 r+s} \\
& \quad \text { from }(1.6 .2 \mathrm{a}) \text { and }(1.4 .3) \\
\equiv & \operatorname{Ad} \varphi\left(Y_{1}\right)\left(Z+\operatorname{ad}\left(Y_{2}\right)(Z)\right) \bmod \mathfrak{g}_{x, 2 r+s} \\
= & \operatorname{Ad} \varphi\left(Y_{1}\right) Z+\operatorname{Ad} \varphi\left(Y_{1}\right)\left(\operatorname{ad}\left(Y_{2}\right)(Z)\right) \\
\equiv & \operatorname{Ad} \varphi\left(Y_{1}\right) Z+\operatorname{ad}\left(Y_{2}\right)(Z) \bmod \mathfrak{g}_{x, 2 r+s} \\
& \quad \text { from }(1.4 .3) \\
\equiv & Z+\operatorname{ad}\left(Y_{1}\right) Z+\operatorname{ad}\left(Y_{2}\right) Z \bmod \mathfrak{g}_{x, 2 r+s} \\
= & Z+\operatorname{ad}\left(Y_{1}+Y_{2}\right) Z .
\end{aligned}
$$

Thus, the proposition holds for all $Y \in \mathfrak{g}_{x, r}$.

Lemma 1.6.4. Suppose $\mathbf{T} \subset \mathbf{G}$ is a maximal F-torus, $x \in \mathcal{A}(T), 0<$ $r \leq s \leq 2 r, X \in \mathfrak{g}_{x, r}$, and $g \in G$. Then

$$
\operatorname{Int}\left(g^{-1}\right) \varphi_{\operatorname{Int}(g) T, g x ; r, s}(g X)=\varphi_{T, x ; r, s}(X) .
$$

Proof. Make the assumptions in (1.6.1). Write $X=X_{0}+\sum_{b \in \Phi(\mathbf{G}, \mathbf{T})} X_{b}$, where $X_{0} \in \mathfrak{t}=\operatorname{Lie}(T)$, and $X_{b} \in \mathfrak{u}_{b}$.

$$
\begin{aligned}
\operatorname{Int}(g) \varphi_{T}(X) & =\operatorname{Int}(g) \varphi_{T}\left(X_{0}+\sum_{b} X_{b}\right) \\
& \equiv \operatorname{Int}(g) \varphi_{T}\left(X_{0}\right) \cdot \prod_{b} \operatorname{Int}(g)\left(\exp _{b} X_{b}\right) \bmod G_{g x, s} \\
& \equiv \varphi_{\operatorname{Int}(g) T}\left(g X_{0}\right) \cdot \prod_{b}\left(\exp _{g b} g X_{b}\right) \bmod G_{g x, s} \\
& =\varphi_{\operatorname{Int}(g) T}(g X)
\end{aligned}
$$

The result follows.

Proposition 1.6.5. Let $\mathbf{T}$ be a maximal $F$-torus in $\mathbf{G}$, and let $x \in \mathcal{A}(T)$. If $0<r \leq s \leq 2 r$, then the map $\varphi_{T, x ; r, s}$ is $G_{x}$-equivariant. 
Proof. Make the assumptions in (1.6.1), and write $\varphi=\varphi_{T}$. It will be sufficient to show that $\varphi_{T, x ; r, s}$ is equivariant under $T_{0}$ and under each affine root group $U_{\psi}$ with $\psi(x) \geq 0$.

Let $g \in T_{0}$. Then for all $Z \in \mathfrak{t}=\operatorname{Lie}(T)$,

$$
\varphi(\operatorname{Ad}(g) Z)=\varphi(Z)=\operatorname{Int}(g) \varphi(Z)
$$

while if $Z \in \mathfrak{u}_{b}$, then

$$
\varphi(\operatorname{Ad}(g) Z)=\varphi(b(g) Z)=\operatorname{Int}(g) \varphi(Z)
$$

since in this case $\varphi$ is just the map $\exp _{b}$.

Now let $g \in U_{\psi}$ with $\psi(x) \geq 0$, and let $Z \in \mathfrak{g}_{x, r}$. Then $g=\varphi(Y)$ for some $Y \in \mathfrak{u}_{\psi}$. We remark that (1.6.2) and (1.6.3) remain valid (with the same proof) for $Y$ and $Z$, even though $Y$ need not lie in $\mathfrak{g}_{x, 0^{+}}$. Therefore,

$$
\begin{array}{rlrl}
\varphi(\operatorname{Ad}(g) Z) & \equiv \varphi(Z+[Y, Z]) \bmod G_{x, 2 r} & & \text { by }(1.6 .3) \\
& \equiv \varphi[Y, Z] \varphi(Z) \bmod G_{x, 2 r} & & \text { by }(1.6 .2 \mathrm{a}) \\
& \equiv[\varphi(Y), \varphi(Z)] \varphi(Z) \bmod G_{x, 2 r} & & \text { by }(1.6 .2 \mathrm{~b}) \\
& =\operatorname{Int}(g) \varphi(Z), &
\end{array}
$$

as desired.

Corollary 1.6.6. The map $\varphi_{T, x ; r, s}$ is independent of the choice of $\mathbf{T}$.

Proof. From (1.4.1), we may assume that $\mathbf{G}$ is $F$-split. Let $\mathbf{T}$ and $\mathbf{T}^{\prime}$ be $F$-split maximal $F$-tori, and suppose $x \in \mathcal{A}\left(T^{\prime}\right) \cap \mathcal{A}(T)$. Then $T=g T^{\prime} g^{-1}$ for some $g \in G_{x}$, so from the proposition and the lemma,

$$
\varphi_{T^{\prime}, x ; r, s}=\operatorname{Int}\left(g^{-1}\right) \circ \varphi_{T, x ; r, s} \circ \operatorname{Ad}(g)=\varphi_{T, x ; r, s} .
$$

From now on, we write $\varphi_{x ; r, s}$ instead of $\varphi_{T, x ; r, s}$, and $\varphi_{x}$ instead of $\varphi_{T, x}$. Although $\varphi_{x}$ is not $G$-equivariant, it has equivariance properties that are almost as useful.

Proposition 1.6.7. Let $g \in G$, and $0<r \leq s \leq 2 r$. If $X, g X \in \mathfrak{g}_{x, r}$, then

$$
\varphi_{x ; r, s}(g X)=\operatorname{Int}(g) \varphi_{x ; r, s}(X)
$$


Proof. From (1.6.4),

$$
\begin{aligned}
\operatorname{Int}\left(g^{-1}\right) \varphi_{g x ; r, s}(g X) & =\varphi_{x ; r, s}(X) \\
\operatorname{Int}\left(g^{-1}\right) \varphi_{x ; r, s}(g X) & =\varphi_{g^{-1} x ; r, s}(X) .
\end{aligned}
$$

Recall the isomorphisms in (1.5.2), and let $S_{1}=\{x, g x\}$ and $S_{2}=\left\{g^{-1} x, x\right\}$. Then

$$
\operatorname{Int}\left(g^{-1}\right) \varphi_{S_{1} ; r, s}(g X)=\varphi_{S_{2} ; r, s}(X)
$$

which implies

$$
\varphi_{S_{1} ; r, s}(g X)=\operatorname{Int}(g) \varphi_{S_{2} ; r, s}(X) .
$$

The result then follows.

1.7. Duality. Recall that $\mathfrak{g}_{x,(-r)^{+}}^{*}=\mathfrak{g}_{x, r}^{\bullet}$. For any $m \leq n$, we have a nondegenerate, $G_{x}$-invariant pairing

$$
\mathfrak{g}_{x,(-n)^{+}}^{*} / \mathfrak{g}_{x,(-m)^{+}}^{*} \times \mathfrak{g}_{x, m} / \mathfrak{g}_{x, n} \longrightarrow \mathbb{C}
$$

given by $(X, Y) \longmapsto \Lambda(X(Y))$. Thus, we have a $G_{x}$-equivariant isomorphism

$$
\mathfrak{g}_{x,(-n)^{+}}^{*} / \mathfrak{g}_{x,(-m)^{+}}^{*} \stackrel{\sim}{\longrightarrow}\left(\mathfrak{g}_{x, m} / \mathfrak{g}_{x, n}\right)^{\widehat{.}} .
$$

Combining this with $\varphi_{x ; m, n}$, we have

$$
\mathfrak{g}_{x,(-n)^{+}}^{*} / \mathfrak{g}_{x,(-m)^{+}}^{*} \stackrel{\sim}{\longrightarrow}\left(G_{x, m} / G_{x, n}\right)^{\wedge}
$$

for all $0<m \leq n \leq 2 m$. Given a coset $\Upsilon=X+\mathfrak{g}_{x,(-m)^{+}}^{*}$ in $\mathfrak{g}_{x,(-n)^{+}}^{*} / \mathfrak{g}_{x,(-m)^{+}}^{*}$, denote the corresponding character by $\chi_{\Upsilon}$ or $\chi_{X}$.

More generally, let $S$ be any group lying between $G_{x, n}$ and $G_{x,(n / 2)^{+}}$. Let $\mathfrak{s}$ be the preimage of $S$ via $\varphi_{x}$. Then the duality map above gives us a correspondence between $\left(S / G_{x, n}\right)^{\wedge}$ and $\mathfrak{g}_{x,(-n)^{+}}^{*} / \mathfrak{s}^{\bullet}$. For a coset $\Upsilon \in$ $\mathfrak{g}_{x,(-n)^{+}}^{*} / \mathfrak{s}^{\bullet}$, let $\chi_{\Upsilon}$ denote the corresponding character of $S / G_{x, n}$.

1.8. Intertwining. Suppose $r>0$, and let $S$ (as above) be any group lying between $G_{x,(r / 2)^{+}}$and $G_{x, r}$. Let $\chi$ be any character of $S / G_{x, r^{+}}$. Then from $\S 1.7, \chi=\chi_{\Upsilon}$ for some coset $\Upsilon \in \mathfrak{g}_{x,-r}^{*} / \mathfrak{s}^{\bullet}$. Let $\mathcal{H}=\mathcal{H}\left(G, \chi_{\Upsilon}\right)$ be the corresponding Hecke algebra of compactly supported, $\chi_{\Upsilon}^{\vee}$-spherical functions on $G$. We have the following analogue of Lemma 3.1 of Chapter 2 in [14].

Lemma 1.8.1. Let $g \in G$. Then $g \in \operatorname{supp} \mathcal{H}\left(G, \chi_{\Upsilon}\right)$ if and only if $g \Upsilon \cap \Upsilon \neq \emptyset$. 
Proof. Let ${ }^{g} \chi_{\Upsilon}$ denote the character on $g S g^{-1}$ that takes $g s g^{-1}$ to $\chi_{\Upsilon}(s)$. Then $g \in \operatorname{supp} \mathcal{H}$ if and only if $\chi_{\Upsilon}^{\vee}={ }^{g} \chi_{\Upsilon}^{\vee}$ (equivalently, $\chi_{\Upsilon}={ }^{g} \chi_{\Upsilon}$ ) on $g S g^{-1} \cap S$. Let $Y \in \Upsilon$. For $s \in g S g^{-1} \cap S$, the following statements are equivalent:

$$
\begin{gathered}
\chi_{\Upsilon}(s)={ }^{g} \chi_{\Upsilon}(s) \\
\chi_{\Upsilon}(s)=\chi_{\Upsilon}\left(g^{-1} s g\right) \\
\Lambda\left(Y\left(\varphi_{x}^{-1}(s)\right)\right)=\Lambda\left(Y\left(\varphi_{x}^{-1}(\operatorname{Int}(g) s)\right)\right) \\
\Lambda\left(Y\left(\varphi_{x}^{-1}(s)\right)\right)=\Lambda\left(Y\left(\operatorname{Ad}(g) \varphi_{x}^{-1}(s)\right)\right) \\
\Lambda\left(Y\left(\varphi_{x}^{-1}(s)\right)\right)=\Lambda\left(\left(g^{-1} Y\right)\left(\varphi_{x}^{-1}(s)\right)\right) \\
\Lambda\left(\left(Y-g^{-1} Y\right)\left(\varphi_{x}^{-1}(s)\right)\right)=0 .
\end{gathered}
$$

The truth of this last statement for all $s \in g S g^{-1} \cap S$ is equivalent to each of the following statements:

$$
\begin{gathered}
Y-g^{-1} Y \in \mathfrak{s}^{\bullet}+g^{-1} \mathfrak{s}^{\bullet} \\
Y+Y_{1}=g^{-1} Y+g^{-1} Y_{2} \quad \text { for some } Y_{1}, Y_{2} \in \mathfrak{s}^{\bullet} \\
g^{-1} \Upsilon \cap \Upsilon \neq \emptyset \\
g \Upsilon \cap \Upsilon \neq \emptyset .
\end{gathered}
$$

1.9. Centralizer subgroups and algebras. Given $X \in \mathfrak{g}$, define

$$
\begin{aligned}
C_{\mathfrak{g}}(X) & =\{Y \in \mathfrak{g} \mid[X, Y]=0\}, \\
C_{\mathbf{G}}(X) & =\{g \in \mathbf{G} \mid g X=X\} .
\end{aligned}
$$

The latter is an $F$-group. Suppose that $X$ is semisimple. Then $C_{\mathfrak{g}}(X)$ is the Lie algebra of $\left(C_{\mathrm{G}}(X)\right)(F)$ (see $\left.[7, \S 1.14]\right)$. If the characteristic of $F$ is not a "torsion" prime for $\mathbf{G}$ in the sense of [36, Definition 2.1], then $C_{\mathbf{G}}(X)$ is connected (see loc. cit., 3.14). If the characteristic of $F$ is not a "bad" prime for $\mathbf{G}$ (in the sense of [35, I.4.1]), then the connected part of $C_{\mathbf{G}}(X)$ is an $E$-Levi subgroup of $\mathbf{G}$ for some Galois extension $E / F$. Starting in (2.1.1), we will exclude all bad and torsion primes for $\mathbf{G}$.

Suppose that $\mathbf{M} \subset \mathbf{G}$ is an $E$-Levi subgroup defined over $F$, let $M=$ $\mathbf{M}(F)$, and $\mathfrak{m}=\operatorname{Lie}(M)$. Since every maximal $E$-split torus in $\mathbf{M}$ is also a maximal $E$-split torus in $\mathbf{G}$, one can embed $\mathcal{B}(\mathbf{M}, E)$ in $\mathcal{B}(\mathbf{G}, E)$, and the set of such embeddings is an affine space. $\operatorname{Gal}(E / F)$ acts on this space, 
and must have a fixed point $i$, a Galois-equivariant embedding. All such embeddings have the same image.

Assume from now on that we can take $E / F$ to be tame. Then, since $\mathcal{B}$ is the $\operatorname{Gal}(E / F)$ fixed point set in $\mathcal{B}(\mathbf{G}, E)$ (see [33, Prop. 5.1.1]), the image of $\mathcal{B}(\mathbf{M}, F)$ lies in $\mathcal{B}$. Given $x \in \mathcal{B}$ lying in this image, we will abuse language and say that $x$ lies in $\mathcal{B}(\mathbf{M}, F)$. Conversely, we will say that any $x \in \mathcal{B}(\mathbf{M}, F)$ also lies in $\mathcal{B}$, even though there may be some ambiguity concerning the image of $x$ in $\mathcal{B}$. This will not cause a problem.

Proposition 1.9.1. For all $x \in \mathcal{B}(\mathbf{M}, F)$,

$$
\begin{aligned}
M \cap G_{x, r} & =M_{x, r}, \\
\mathfrak{m} \cap \mathfrak{g}_{x, r} & =\mathfrak{m}_{x, r}, \\
\mathfrak{m}^{*} \cap \mathfrak{g}_{x, r}^{*} & =\mathfrak{m}_{x, r}^{*} .
\end{aligned}
$$

Proof. From (1.4.1), we may assume that $\mathbf{G}$ is $F$-split, and that $\mathbf{M}$ is an $F$ Levi subgroup. Then the compatibility of the filtrations is clear from the fact that $M_{i(x), r}$, etc., is independent of the choice of embedding $i: \mathcal{B}(\mathbf{M}, F) \longrightarrow$ $\mathcal{B}$.

Proposition 1.9.2. For all $x \in \mathcal{B}(\mathbf{M}, F)$ and all $0<r \leq s \leq 2 r$,

$$
\varphi_{x ; r, s}^{M}=\left.\varphi_{x ; r, s}\right|_{\mathfrak{m}_{x, r} / \mathfrak{m}_{x, s}},
$$

where $\varphi_{x}^{M}$ is a mock exponential map for $M$.

Proof. From (1.4.1), we may assume that $x \in \mathcal{A}(T)$, where $\mathbf{T}$ is an $F$-split maximal torus in $\mathbf{M}$. Then the result is clear from (1.6.6).

If there exists an $F$-valued, $G$-invariant, nondegenerate, symmetric, bilinear form $B$ on $\mathfrak{g}$, then, after fixing a choice of $B$, let $\mathfrak{m}^{\perp}$ denote the perpendicular of $\mathfrak{m}$ in $\mathfrak{g}$ with respect to $B$. For $x \in \mathcal{B}(\mathbf{M}, F)$ and $s \in \mathbb{R}$, let $\mathfrak{m}_{x, s}^{\perp}$ denote $\mathfrak{m}^{\perp} \cap \mathfrak{g}_{x, s}$.

Proposition 1.9.3. If $x \in \mathcal{B}(\mathbf{M}, F)$ and $s \in \mathbb{R}$, then $\mathfrak{g}_{x, s}=\mathfrak{m}_{x, s} \oplus \mathfrak{m}_{x, s}^{\perp}$.

Proof. In view of (1.4.1), we may assume that $\mathfrak{m}$ is a Levi subalgebra of $\mathfrak{g}$. Then the result is clear from the definition of the filtration and from fact that $x$ lies in the apartment of some torus in $\mathbf{M}$.

Remark 1.9.4. Suppose $F$ has characteristic zero, $p=2, \mathbf{G}=\mathbf{G L}_{2}$, and $\mathbf{M}=\left\{\left(\begin{array}{cc}a & b \\ \varpi b & a\end{array}\right) \mid a^{2}-\varpi b^{2} \neq 0\right\}$. Then the usual Iwahori filtration provides an apparent counterexample to (1.9.3). However, it turns out that this filtration corresponds to a point $x$ that does not lie in $\mathcal{B}(\mathbf{M}, F)$. 
Imitating Moy and Prasad [27], we will call a coset in $\mathfrak{g}$ nondegenerate if it contains no nilpotent elements.

Lemma 1.9.5. Suppose $X \in \mathfrak{g}$ is semisimple, $C_{\mathrm{G}}(X)$ is an $F^{\prime}$-Levi subgroup of $\mathbf{G}$ for some tame $F^{\prime} / F, x \in \mathcal{B}\left(C_{\mathbf{G}}(X), F\right)$, and $X \in \mathfrak{g}_{x,-r} \backslash \mathfrak{g}_{x,(-r)^{+}}$. Then the coset $X+\mathfrak{g}_{x,(-r)}+$ is nondegenerate.

Proof. Let $E$ be a field extension of $F^{\prime}$ over which $C_{\mathbf{G}}(X)$ splits, and let $e$ be the ramification degree of $E / F$. Then $X \in \mathfrak{g}(E)_{x,-e r} \backslash \mathfrak{g}(E)_{x,(-e r)^{+}}$, and $x$ lies in the apartment of some $E$-split maximal torus whose Lie algebra contains $X$. Thus er $\in \mathbb{Z}$. As modules over $\mathbf{G}(E)_{x} / \mathbf{G}(E)_{x, 0^{+}}$,

$$
\mathfrak{g}(E)_{x,-e r} / \mathfrak{g}(E)_{x,(-e r)^{+}} \cong \mathfrak{g}(E)_{x, 0} / \mathfrak{g}(E)_{x, 0^{+}} \cong \operatorname{Lie}\left(\mathbf{G}(E)_{x} / \mathbf{G}(E)_{x, 0^{+}}\right) .
$$

The image $\bar{X}$ of $X$ in the rightmost space is clearly not nilpotent. By [27, Prop. 4.3] and its proof, $X+\mathfrak{g}(E)_{x,(-e r)^{+}}$is nondegenerate. Therefore, so is $X+\mathfrak{g}_{x,(-r)^{+}}$.

\section{Refinement.}

2.1. A tameness hypothesis and its consequences. Recall that any nondegenerate $G$-invariant symmetric bilinear form $B$ on $\mathfrak{g}$ induces a $G$ equivariant identification

$$
\Psi: \mathfrak{g} \longrightarrow \mathfrak{g}^{*} .
$$

For any extension $E / F$, we will also denote the induced map $\mathfrak{g}(E) \longrightarrow \mathfrak{g}^{*}(E)$ by $\Psi$. If $\mathbf{T} \subset \mathbf{G}$ is an $E$-split maximal $F$-torus, and $b \in \Phi(\mathbf{G}, \mathbf{T})$, then let $\mathfrak{u}_{b}^{*}$ denote the subspace of $\mathfrak{g}^{*}(E)$ that transforms according to the root $b$ under the action of $\mathbf{T}(E)$. In particular, $\mathfrak{u}_{b}^{*}$ is the dual of $\mathfrak{u}_{-b}$, not of $\mathfrak{u}_{b}$. Then it is clear from the definitions that $\Psi\left(\mathfrak{u}_{b}\right)=\mathfrak{u}_{b}^{*}$.

From now on, we make the following assumption:

Hypothesis 2.1.1. The residual characteristic $p$ of $F$ is odd and does not divide the order of the center of the simply connected cover of $\mathcal{D} \mathbf{G}$. Moreover, if $\mathbf{G}$ has a simple factor of type $G_{2}$, then $p \neq 3$. If $F$ has positive characteristic $p$, then $p>3$ if $\mathbf{G}$ contains any exceptional simple factors, and $p>5$ if $\mathbf{G}$ contains any factors of type $E_{8}$.

As a consequence, not only does such a form $B$ exist, but we can (and will) choose it so that for all $x \in \mathcal{B}$ and all $s \in \mathbb{R}$, the corresponding isomorphism $\Psi$ takes $\mathfrak{g}_{x, s}$ to $\mathfrak{g}_{x, s}^{*}$. A special case of this is proved in [32]; see [2] for a proof under slightly more general hypotheses than (2.1.1). 
From now on, use $\Psi$ to identify $\mathfrak{g}$ and $\mathfrak{g}^{*}$.

Proposition 2.1.2. For all $x \in \mathcal{B}$ and for all $s \in \mathbb{R}$,

$$
\mathfrak{g}_{x, s}=\left(\mathcal{Z} \mathfrak{g} \cap \mathfrak{g}_{x, s}\right) \oplus\left(\mathcal{D} \mathfrak{g} \cap \mathfrak{g}_{x, s}\right) .
$$

Proof. As usual, we may assume that $\mathbf{G}$ is $F$-split. Choose a maximal $F$-split $F$-torus $\mathbf{T}$ in $\mathbf{G}$ with root system $\Phi$, and let $\mathfrak{t}=\operatorname{Lie}(\mathbf{T}(F))$. Let $Z \in \mathcal{Z} \mathfrak{g} \cap \mathfrak{t}_{s}$ and $Y \in \mathcal{D} \mathfrak{g} \cap \mathfrak{t}_{s}$. Assume that at least one of $Y$ and $Z$ does not lie in $\mathfrak{t}_{s+1}$. From (1.9.3), it is enough to show that $Z+Y$ does not lie in $\mathfrak{t}_{s+1}$. First, assume that $Y \notin \mathfrak{t}_{s+1}$. From (2.1.1), $p$ does not divide

$$
\left|\mathbf{X}^{*}(\mathbf{T}) /\left(\mathbf{X}_{0}+\mathbb{Z} \Phi\right)\right|
$$

where $\mathbf{X}_{0}=\left\{\chi \in \mathbf{X}^{*}(\mathbf{T}) \mid\left\langle b^{\vee}, \chi\right\rangle=0\right.$ for all $\left.b \in \Phi\right\}$. Therefore, there is a root $b \in \Phi$ such that $\nu(d b(Y))=s$. Since $d b(Z+Y)=d b(Y), Z+Y \notin \mathfrak{t}_{s+1}$. Now assume that $Z \notin \mathfrak{t}_{s+1}$. Then for some $\chi \in \mathbf{X}_{0}, s=\nu(d \chi(Z))=$ $\nu(d \chi(Z+Y))$.

This result actually holds under slightly weaker hypotheses than (2.1.1). See [2] for details.

\subsection{Good cosets and good elements.}

Definition 2.2.3. Say that a set $\Upsilon \subset \mathfrak{g}$ is $\operatorname{good}$ if $\Upsilon \in \mathfrak{g}_{x, r} / \mathfrak{g}_{x, r}$ for some $x \in \mathcal{B}$ and some $r \in \mathbb{R}$, and there is some maximal $F$-torus $\mathbf{T} \subset \mathbf{G}$ such that Lie $(\mathbf{T}(F))$ intersects $\Upsilon$, $\mathbf{T}$ splits over a tamely ramified extension $E$ of $F$, and $x$ belongs to the apartment of $\mathbf{T}(E)$ in $\mathcal{B}(\mathbf{G}, E)$.

It is clear from the definition that any semisimple $X$ that lies in the Lie algebra of a tamely ramified Cartan subalgebra lies in some good coset. From (1.9.5), any good coset is nondegenerate. Call an unrefined minimal Ktype of positive depth good if it corresponds to a good coset in $\mathfrak{g}$. As remarked in the introduction, smooth representations of positive depth generally seem to contain good K-types, at least in the absence of wild ramification.

Suppose $X \in \mathfrak{g}$ belongs to $\mathfrak{t}=\mathfrak{t}(F)$, where $\mathfrak{t}$ is the Lie algebra of some $F$-split maximal torus $\mathbf{T}$. Then there is some $r \in \mathbb{Z}$ such that $X \in \mathfrak{t}_{r} \backslash \mathfrak{t}_{r+1}$. Call $X$ a $\mathbf{T}$-good element of depth $r$ if for every root $\alpha$ of $\mathbf{G}$ with respect to $\mathbf{T}, d \alpha(X)$ either is zero or has valuation $r$.

Suppose $\mathbf{T}^{\prime}$ is another $F$-split maximal torus with Lie algebra $\mathfrak{t}^{\prime}$, and $X \in \mathfrak{t} \cap \mathfrak{t}^{\prime}$. Then there is some $g \in G$ such that $g X=X$ and $g \mathfrak{t}=\mathfrak{t}^{\prime}$. This implies that $X$ is $\mathbf{T}$-good if and only if it is $\mathbf{T}^{\prime}$-good, and its depth is independent of the choice of split torus. 
Suppose that $\mathbf{T}$ is an arbitrary maximal $F$-torus, split over some tame Galois extension $E / F$. Clearly, $\operatorname{Gal}(E / F)$ preserves the set of $\mathbf{T}$-good elements of $\mathfrak{t}(E)$ of any given depth. This allows us to make the following definition.

Definition 2.2.4. A semisimple element $X \in \mathfrak{g}$ is good if for some (hence any) tamely ramified $F$-torus $\mathbf{T}$ satisfying $X \in \mathfrak{t}$, where $\mathfrak{t}=\operatorname{Lie}(\mathbf{T})$, and some (hence any) $E / F$ over which $\mathbf{T}$ splits, for every root $\alpha$ of $\mathbf{G}$ with respect to $\mathbf{T}, d \alpha(X)$ either is zero or has $E$-normalized valuation $r$, where $X \in \mathfrak{t}(E)_{r} \backslash \mathfrak{t}(E)_{r+1}$. If this is also true whenever $\alpha$ is a sum of two roots, then we will call $X$ very good.

Note that the set of good elements is preserved by the adjoint action of $G$.

While good cosets will typically contain good elements, one can cook up some counterexamples. Therefore, it is useful to know under what circumstances one can guarantee the existence of good elements.

Proposition 2.2.5. Let $\mathbf{T}$ be a tamely ramified maximal $F$-torus in $\mathbf{G}$, and let $\mathfrak{t}=\operatorname{Lie}(\mathbf{T}(F))$. For every $r \in \mathbb{R}$, every coset in $\mathfrak{t}_{r} / \mathfrak{t}_{r}+$ contains a good element provided that $\mathbf{G}$ contains no simple factors of exceptional type. Otherwise, we require that $p>\mathrm{c}(\Phi)$ for every exceptional simple root system $\Phi$ occurring as a factor in $\Phi(\mathbf{G}, \mathbf{T})$, where $\mathrm{c}(\Phi)$ is a constant.

For example, $\mathrm{c}\left(\mathrm{G}_{2}\right)=7$. Note that we are still assuming (2.1.1). For a proof of the proposition (under hypotheses that are weaker, but more complicated to state), see [2]. One can also find there a proof that, provided $p$ is large enough, every coset in $\mathfrak{t}_{r} / \mathfrak{t}_{r}+$ contains a very good element.

2.3. Slightly refined minimal K-types. From now on, let $r>0$, and let $X+\mathfrak{g}_{x,(-r)^{+}} \in \mathfrak{g}_{x,-r} / \mathfrak{g}_{x,(-r)^{+}}$be a good coset, and $X$ a good element such that $x \in \mathcal{B}(\mathbf{M}, F)$, where $\mathbf{M}=C_{\mathbf{G}}(X)$. Let $M=\mathbf{M}(F)$ and $\mathfrak{m}=\operatorname{Lie}(M)$.

Lemma 2.3.1. The map

$$
\operatorname{ad}(X): \mathfrak{m}_{x, s}^{\perp} / \mathfrak{m}_{x, s^{+}}^{\perp} \longrightarrow \mathfrak{m}_{x,-r+s}^{\perp} / \mathfrak{m}_{x,(-r+s)^{+}}^{\perp}
$$

is an isomorphism.

Proof. In view of (1.4.1), we may assume that $\mathbf{M}$ is $F$-split, in which case the result follows directly from the fact that $X$ is good.

Lemma 2.3.2. Let $Y \in X+\mathfrak{g}_{x,(-r)^{+}}$, and suppose that $Y \in \mathfrak{m}+\mathfrak{g}_{x, s}$ for some $s>-r$. Then $Y$ is conjugate under $G_{x, s+r}$ to an element of $X+\mathfrak{m}_{x,(-r)^{+}}$. 
Proof. (This is adapted from [26, Lemma 4.4].) By induction, it will be enough to show that $Y$ is $G_{x, s+r}$-conjugate to an element of $X+\mathfrak{m}_{x,(-r)^{+}}+$ $\mathfrak{g}_{x, s^{+}}$.

From (1.9.3), we may write $Y=Y_{1}+Y^{\perp}$, with $Y_{1} \in X+\mathfrak{m}_{x,(-r)^{+}}$and $Y^{\perp} \in \mathfrak{m}_{x, s}^{\perp}$. From (2.3.1), there exists $Z^{\perp} \in \mathfrak{m}_{x, s+r}^{\perp}$ such that $\operatorname{ad}(Y)\left(Z^{\perp}\right) \equiv$ $Y^{\perp} \bmod \mathfrak{g}_{x, s^{+}}$. So, from (1.6.3),

$$
\operatorname{Ad}\left(\varphi_{x}\left(Z^{\perp}\right)\right)(Y) \equiv Y-\operatorname{ad}(Y)\left(Z^{\perp}\right) \equiv Y_{1} \bmod \mathfrak{g}_{x, s^{+}} .
$$

Define

$$
\begin{aligned}
\mathfrak{J}_{+} & =\mathfrak{m}_{x, r} \oplus \mathfrak{m}_{x,(r / 2)^{+}}^{\perp} \\
\mathfrak{J} & =\mathfrak{m}_{x, r} \oplus \mathfrak{m}_{x,(r / 2)}^{\perp} .
\end{aligned}
$$

Let $J_{+}=\varphi_{x}\left(\mathfrak{J}_{+}\right)$and $J=\varphi_{x}(\mathfrak{J})$. These are groups. Via the bijection $\varphi_{x}$, one may regard the character $\chi=\chi_{X}$ as being defined on $\mathfrak{g}_{x, r} / \mathfrak{g}_{x, r+}$. Since $\chi$ is trivial on $\mathfrak{m}_{x, r}^{\perp}$, we may extend it to a character $\bar{\chi}$ of $\mathfrak{J}_{+}$by letting it be trivial on $\mathfrak{m}_{x,(r / 2)^{+}}^{\perp}$.

Lemma 2.3.3 All extensions of $\chi$ to $\mathfrak{J}_{+}$are $G_{x}$-conjugate to $\bar{\chi}$.

Proof. From $\S 1.7$, characters of $\mathfrak{J}_{+} / \mathfrak{g}_{x, r^{+}}$correspond to cosets in $\mathfrak{g}_{x,-r} / \mathfrak{J}_{+}^{\bullet}$, where $\mathfrak{J}_{+}^{\bullet}=\mathfrak{m}_{x,(-r)^{+}} \oplus \mathfrak{m}_{x,-r / 2}^{\perp}$. Let $\chi^{\prime}$ be an extension of $\chi$ to $\mathfrak{J}_{+}$. Then $\chi^{\prime}$ is given by the formula

$$
\chi^{\prime}(Y)=\Lambda\left(B\left(X+X_{1}, Y\right)\right)
$$

where $X_{1} \in \mathfrak{m}_{x,-r / 2}^{\perp}$. From (2.3.2), there is some $g \in G_{x, r / 2}$ such that $\operatorname{Ad}(g)\left(X+X_{1}\right) \equiv X \bmod \mathfrak{m}_{x,(-r)^{+}}$. Thus, for all $Y \in \mathfrak{J}_{+}$,

$$
\chi^{\prime}\left(g^{-1} Y\right)=\Lambda\left(B\left(X+X_{1}, g^{-1} Y\right)\right)=\Lambda(B(X, Y))=\bar{\chi}(Y) .
$$

Now regard $\bar{\chi}$ as a character of $J_{+}$.

Corollary 2.3.4. Any admissible representation of $G$ that contains $\left(G_{x, r}, \chi\right)$ also contains $\left(J_{+}, \bar{\chi}\right)$.

Proposition 2.3.5. $\quad \operatorname{supp} \mathcal{H}(G, \bar{\chi}) \subseteq J M J$. 
Proof. See [2] for a general proof. The following works if we assume that $X$ is very good (as we may if $p$ is large enough). Suppose $g \in \operatorname{supp} \mathcal{H}(G, \bar{\chi})$. From (1.8.1), there exist $Y_{1}, Y_{2} \in \mathfrak{g}_{x,-r}$ such that $Y_{i} \equiv X \bmod \mathfrak{J}_{+}^{\bullet}$ and $g Y_{1}=$ $Y_{2}$. From (2.3.2), we can find $Z_{1}, Z_{2} \in \mathfrak{m}_{x,-r}$ such that $Z_{i} \equiv X \bmod \mathfrak{g}_{x,(-r)^{+}}$ and $Y_{i}=k_{i} Z_{i}$, for some $k_{i} \in G_{x, 0^{+}}$. By density, we may take the $Y_{i}$, and thus the $Z_{i}$, to be regular and semisimple.

Let $g^{\prime}=k_{2}^{-1} g k_{1}$. Then $g^{\prime} Z_{1}=Z_{2}$, so $g^{\prime} \mathfrak{t}^{(1)}=\mathfrak{t}^{(2)}$, where $\mathfrak{t}^{(i)}$ is the unique Cartan subalgebra containing $Z_{i}$. Both $X$ and $g^{\prime} X$ are very good elements that lie in $\mathfrak{t}^{(2)}$, and they lie in the same coset modulo $\mathfrak{t}_{(-r)^{+}}^{(2)}$. Since they are conjugates, they must have the same multisets of eigenvalues under the adjoint representation. By the definition of very good, they are equal. Thus, $g^{\prime} \in M$, so $g \in G_{x, r / 2} M G_{x, r / 2}=J M J$.

2.4. Anisotropic case. From now on, suppose that $M / \mathcal{Z} G$ is compact. Then $\mathcal{B}(\mathbf{M}, F)=\left\{x+\lambda \mid \lambda \in \mathbf{X}_{*}(\mathbf{S}) \otimes \mathbb{R}\right\}$, where $\mathbf{S}$ is the $F$-split part of $\mathcal{Z} \mathbf{G}$.

Proposition 2.4.1. $\quad M$ normalizes $\mathfrak{g}_{x, r}($ for all $s \in \mathbb{R})$ and $G_{x, s}$ (for all $s \geq 0)$.

Proof. This follows from the fact that $M$ acts on $\mathcal{B}(\mathbf{M}, F)$, and for all $\lambda \in$ $\mathbf{X}_{*}(\mathbf{S}) \otimes \mathbb{R}, G_{x, s}=G_{x+\lambda, s}$ and $\mathfrak{g}_{x, s}=\mathfrak{g}_{x+\lambda, s}$.

Lemma 2.4.2. $\quad \mathfrak{m}^{\perp}$ is an $M$-module.

Proof. Let $g \in M, Z \in \mathfrak{m}^{\perp}$. Then $B(g Z, \mathfrak{m})=B\left(Z, g^{-1} \mathfrak{m}\right)=0$, so $g Z \in$ $\mathfrak{m}^{\perp}$.

Proposition 2.4.3. $\quad M$ normalizes $J$ and $J_{+}$.

Proof. This follows from the lemma, (2.4.1), and (1.6.7).

2.5. Supercuspidal representations. Recall that the support of $\mathcal{H}(G, \bar{\chi})$ lies in $J M J=M J$. Let $\sigma$ be any representation of $M J$ that contains $\bar{\chi}$. We shall see (2.6.3) that $\sigma$ must in fact restrict to a multiple of $\bar{\chi}$. Thus, $\operatorname{supp} \mathcal{H}(G, \sigma) \subseteq M J$, so $\mathcal{H}(G, \sigma) \cong \mathbb{C}$.

Let $\pi_{\sigma}=\operatorname{Ind}_{M J}^{G} \sigma$ be the compactly induced representation of $G$. Then

$$
\operatorname{End}_{G}\left(\pi_{\sigma}\right) \cong \mathcal{H}(G, \sigma) \cong \mathbb{C}
$$

so $\pi_{\sigma}$ is irreducible. It is supercuspidal by [8, Theorem 5.3.1]. 
Conversely, if $\pi$ is any irreducible representation of $G$ containing $\sigma$, then Frobenius reciprocity implies that $\pi$ is equivalent to $\pi_{\sigma}$, and thus is supercuspidal.

This proves the following result:

Theorem 2.5.1. Suppose that $\chi$ is an anisotropic unrefined minimal Ktype for $G$, and assume that the corresponding coset is good and contains a good element. Assume further that the hypotheses of (2.1.1) are satisfied, and that $M$ and $J$ are defined as above. Then every representation of $G$ that contains $\chi$ is induced from a representation of $M J$ that contains $\chi$.

2.6. Inducing data. We now construct irreducible representations of $M J$ containing $\left(J_{+}, \bar{\chi}\right)$, and show that such representations are in bijection with the representations of $M$ containing $\left(M_{x, r}, \chi\right)$. (Our method goes back to Gérardin [11] and Howe [12], though Carayol's variant [5, $\S \S 4.2-5.4]$ can be modified to work in the present context if we restrict our attention to one-dimensional representations of $M$.) Let $N=\operatorname{ker} \bar{\chi}$.

First, look at the special case where $J=J_{+}$. Then $M J / N \cong M /(N \cap M)$, and the result is clear.

More generally, $J$ and $J_{+}$need not be equal. Let $A=J_{+} / N$ and let $H=J / N$. For $a, b \in H$, let $\langle a, b\rangle=\bar{\chi}([a, b])$. Since $A$ is central in $H$, $\langle a, b\rangle$ depends only on the class of $a$ and $b$ modulo $A$. Thus, $\langle$,$\rangle is an$ alternating form on $H / A \cong J / J_{+}$.

Lemma 2.6.1. The form $\langle$,$\rangle is nondegenerate on J / J_{+}$.

Proof. Let $a, b \in J$, and assume that $a \notin J_{+}$. Write $a=\varphi_{x}(Y)$ and $b=$ $\varphi_{x}(Z)$. Multiplying $a$ and $b$ by elements of $J_{+}$, we may arrange matters so that $Y, Z \in \mathfrak{m}_{x, r / 2}^{\perp}$, and $Y \notin \mathfrak{m}_{x,(r / 2)^{+}}^{\perp}$. From (1.6.2b), $[a, b] \equiv \varphi_{x}[Y, Z] \bmod$ $G_{x, r^{+}}$, so

$$
\langle a, b\rangle=\Lambda(B(X,[Y, Z]))=\Lambda(B([X, Y], Z)) .
$$

By (2.3.1), $[X, Y] \in \mathfrak{m}_{x,-r / 2}^{\perp} \backslash \mathfrak{m}_{x,(-r / 2)^{+}}^{\perp}$.

It will be enough to show that there exists a choice of $b \in J$ (and thus of $\left.Z \in \mathfrak{m}_{x, r / 2}^{\perp}\right)$ such that $\Lambda(B([X, Y], Z)) \neq 1$. Suppose that there is no such $Z$. Then $B\left([X, Y], \mathfrak{g}_{x, r / 2}\right)=B\left([X, Y], \mathfrak{m}_{x, r / 2}^{\perp}\right) \subset \varpi \mathcal{O}$, so that $[X, Y] \in \mathfrak{g}_{x, r / 2}^{\bullet}=$ $\mathfrak{g}_{x,(-r / 2)^{+}}$, a contradiction.

Consequently, $J / J_{+}$with the form $\langle$,$\rangle is a symplectic space over the$ residue field of $F$, and $H$ is a Heisenberg group. The representation theory of such groups is well known (for example, see [11, Lemma 1.2]). It follows immediately that there exists a unique (up to equivalence) irreducible representation $\rho=\rho_{\chi}$ of $J$ whose restriction to $J_{+}$contains $\bar{\chi}$. In fact, $\left.\rho\right|_{J_{+}}$is a direct sum of $\sqrt{\left|J / J_{+}\right|}$copies of $\bar{\chi}$.

Lemma 2.6.2. $\quad M$ acts on $J / J_{+}$, preserving the form $\langle$,$\rangle .$ 
Proof. That $M$ acts on $J / J_{+}$follows from (2.4.3). Let $a, b \in J$, and let $m \in M$. Then

$$
\begin{aligned}
\langle\operatorname{Int}(m) a, \operatorname{Int}(m) b\rangle & =\Lambda\left(B\left(X, \varphi_{x}^{-1}(\operatorname{Int}(m)[a, b])\right)\right) \\
& =\Lambda\left(B\left(X, m \varphi_{x}^{-1}([a, b])\right)\right) \quad \text { from (1.6.7) } \\
& =\Lambda\left(B\left(m X, \varphi_{x}^{-1}([a, b])\right)\right) \\
& =\Lambda\left(B\left(X, \varphi_{x}^{-1}([a, b])\right)\right)=\langle a, b\rangle .
\end{aligned}
$$

Let $\mathrm{Sp}$ be the symplectic group of $\left(J / J_{+},\langle\rangle,\right)$. From the Lemma, we have a map $M \longrightarrow$ Sp. The theory of the oscillator representation (as presented, for example, in [11, Theorem 2.4]), picks out a unique extension of $\rho_{\chi}$ to a representation $\omega_{\chi}$ of $\operatorname{Sp} \ltimes J$. Let $\omega_{\chi}$ also denote the pullback to $M \ltimes J$. It is clear that the irreducible representations of $M$ extend trivially to $M \ltimes J$. Such representations are in bijection with the irreducible representations of $M \ltimes J$ containing $\rho_{\chi}$, via the map $\sigma \longmapsto \sigma \otimes \omega_{\chi}$. The tensor product $\sigma \otimes \omega_{\chi}$ gives a representation of $M J$ if and only if $\left.\sigma\right|_{M_{x, r}}$ is a multiple of $\left.\chi\right|_{M_{x, r}}$. All irreducible representations of $M J$ containing $\bar{\chi}$ arise in this way. This proves the following:

Proposition 2.6.3. $\quad$ The irreducible smooth representations of $M J$ that contain $\left(J_{+}, \bar{\chi}\right)$ all restrict to multiples of $\bar{\chi}$, and are naturally parametrized by the irreducible smooth representations of $M$ that contain $\left(M_{x, r}, \chi\right)$. If $\sigma$ is such a representation of $M$, then the corresponding representation of $M J$ has dimension $\operatorname{dim}(\sigma) \cdot \sqrt{\mid \mathfrak{m}_{x,(r / 2)}^{\perp} / \mathfrak{m}_{x,(r / 2)^{+}}^{\perp}}$.

2.7. Hecke algebra transfer. Suppose $\chi$ is as in (2.5.1). Then (2.5.1), (2.6.3), and (2.3.4) together imply the following:

Theorem 2.7.1. The irreducible smooth representations of $G$ that contain $\left(G_{x, r}, \chi\right)$ naturally correspond to the irreducible smooth representations of $M$ that contain $\left(M_{x, r}, \chi\right)$.

We can also see this correspondence at the level of Hecke algebras. As before, let $\rho=\rho_{\chi}$ denote the (unique up to isomorphism) Heisenberg extension of $\bar{\chi}$ from $J_{+}$to $J$. From (2.3.4), the irreducible smooth representations of $G$ that contain $\left(G_{x, r}, \chi\right)$ contain $(J, \rho)$, and thus correspond to simple $\mathcal{H}(G, \rho)$-modules. From (2.3.5) and (2.4.3),

$$
\operatorname{supp} \mathcal{H}(G, \rho) \subseteq \operatorname{supp}(G, \bar{\chi})=J M J=M J,
$$


so restriction of functions induces isomorphisms

$$
\mathcal{H}(G, \rho) \stackrel{\sim}{\longrightarrow} \mathcal{H}(M J, \rho) \stackrel{\sim}{\longrightarrow} \mathcal{H}(M, \chi) \otimes_{\mathbb{C}} \operatorname{End}_{\mathbb{C}}\left(\rho^{\vee}\right) .
$$

This last algebra is Morita equivalent [18] to $\mathcal{H}(M, \chi)$, whose simple modules correspond to irreducible smooth representations of $M$ containing $\left(M_{x, r}, \chi\right)$.

2.8. Refined anisotropic K-types. Let $\left[G, \pi_{\sigma}\right]$ denote the inertial equivalence class of $\pi_{\sigma}$, that is, the class of all representations $\pi$ of $G$ such that $\pi \simeq \pi_{\sigma} \otimes \eta$ for some unramified character $\eta$ of $G$. We want to find a compact open subgroup $R \subset G$ and a representation $\rho$ of $R$ such that $(R, \rho)$ is a $\left[G, \pi_{\sigma}\right]$-type in the sense of Bushnell-Kutzko [4]. That is, any smooth irreducible representation $\pi$ of $G$ contains $(R, \rho)$ if and only if $\pi$ belongs to $\left[G, \pi_{\sigma}\right]$.

It is clear (from [4, Prop. 5.4]) that one can take $R$ to be $\operatorname{stab}_{M}(x) J_{+}$ (which contains $M_{x} J_{+}$with finite abelian quotient), and $\rho$ to be an irreducible constituent of $\left.\sigma\right|_{R}$. In case $\sigma$ is parametrized by a representation $\sigma^{\prime}$ of $M$ of dimension greater than 1 , it is possible to replace $R$ by a smaller group $R^{\prime}$ related to the inducing subgroup for $\sigma^{\prime}$. Details will appear elsewhere.

\section{Examples.}

Given an anisotropic $X \in \mathfrak{g}$ that splits over a tame extension, one can use the preceding to find a family of supercuspidal representations of $G$. In case $X$ is singular, some of these representations will be singular, i.e. not associated to any maximal torus in $G$. Below, we give some examples of such elements.

3.1. Symplectic groups. Assume that the residual characteristic of $F$ is not 2 . Let $G$ be a symplectic group in $2 n$ variables, with $n>1$. Realize $G$ as the matrices preserving the bilinear form on $F^{2 n}$ given by the matrix

$$
\left(\begin{array}{rr}
0 & N \\
-N & 0
\end{array}\right)
$$

where $N$ is the $n$-by- $n$ matrix

$$
\left(\begin{array}{lllll} 
& & & & \\
& & & 1 & \\
& & & \therefore & \\
& & & & \\
& & & & \\
1 & & &
\end{array}\right) \text {. }
$$


Let $\varepsilon$ be a nonsquare unit in $F$. Let $X$ be an element of the Lie algebra such that $\varpi^{r} X$ has one of the following forms:

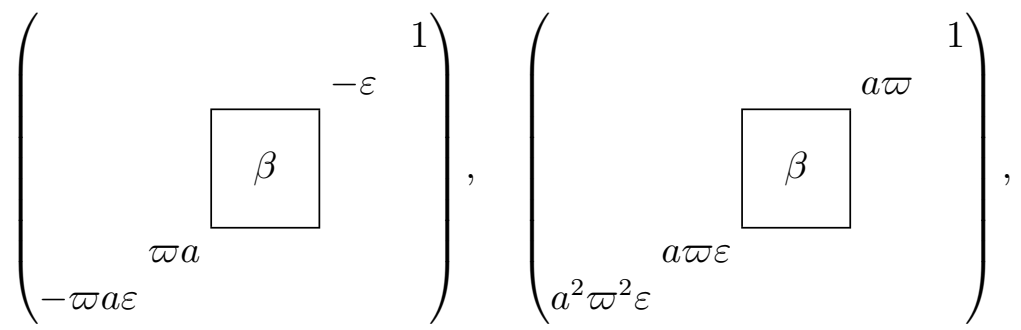

where $\beta$ is a regular element of an elliptic Cartan subgroup of $\operatorname{Sp}_{2(n-2)}(F)$ and $a \in \mathcal{O}^{\times}$. (These are suggested by cases (5.13i.1) and (5.1e.2) in [26].) Then $X$ is singular and anisotropic. We can find many more singular anisotropic elements by combining these two examples using the methods of $\S 3.2$.

3.2. Special orthogonal groups. We show that, with a few low-rank exceptions, all special orthogonal groups (split or not) contain singular anisotropic elements.

Assume that $F$ has odd residual characteristic. Any nondegenerate quadratic form over $F$ is determined up to equivalence by its dimension, its discriminant (modulo squares), and its Hasse invariant. If two forms differ by a scalar multiple, then they give rise to the same orthogonal group and Lie algebra. The usual split form in dimension $n$ has discriminant $(-1)^{\left(\begin{array}{l}n \\ 2\end{array}\right)}$ and Hasse invariant 1 . Let $V_{0}$ be the usual anisotropic space of dimension 4, which has discriminant 1 and Hasse invariant -1 .

Suppose a vector space $V$ over $F$ is equipped with a nondegenerate quadratic form with matrix $J$. Then $\operatorname{End}(V)$ has a natural anti-involution

$$
\sigma(x)=J^{-1 t} x J
$$

Let $G_{0}(V)$ be the set of endomorphisms $x$ such that $x \cdot \sigma(x)=1$. This is the orthogonal group of $(V, J)$. Let $G=G(V)$ be the corresponding special orthogonal group.

Suppose we write $V$ as the orthogonal direct sum $V_{1} \oplus \cdots \oplus V_{r}$, where each summand has even dimension, with at most one exception.

Let $T_{i}$ be a maximal anisotropic elliptic torus in $G\left(V_{i}\right)$. Then, from work of Morris [21], $T=T_{1} \times \cdots \times T_{r}$ is a maximal elliptic torus in $G$. Choose an element $c=\left(c_{1}, \ldots, c_{r}\right)$ of $T$ such that the sets of eigenvalues of the actions of each $c_{i}$ on $V_{i}$ are disjoint. Then we claim that the centralizer $C_{G}(c)$ of $c$ in $G$ is contained in $G\left(V_{1}\right) \times \cdots \times G\left(V_{r}\right)$. For let $S$ be a minimal torus in $T$ containing c. $V$ has a unique decomposition (a refinement of the one already given) into irreducible $S$-modules. Let $W$ be any irreducible $S$-submodule 
of, say, $V_{\ell}$. Let $g \in C_{G}(c)$. Then $\left.c\right|_{W}$ acts with the same eigenvalues as $\left.g c g^{-1}\right|_{g W}$, which is equal to $\left.c\right|_{g W}$. By our choice of $c, g W$ must lie in $V_{\ell}$. This proves the claim.

Suppose that one of the summands of $V$ (say $V_{1}$ ) is isomorphic to a fourdimensional anisotropic space (such as $V_{0}$ ). Then we may clearly choose $c$ so that $c_{i}$ is regular in $G\left(V_{i}\right)$ for $i>1$, and $c_{1}$ lies in some one-dimensional torus in $G\left(V_{1}\right)$. In this case, the centralizer of $c$ will be a product of $C_{G\left(V_{1}\right)}\left(c_{1}\right)$ and a collection of elliptic tori. The former will be a compact, nonabelian group. This leads to the following result:

Proposition 3.2.1. $\quad \mathfrak{s o}(V)$ has singular elliptic elements if an anisotropic space of dimension four is an orthogonal direct summand of $V$.

Which spaces $V$ can be written as such a sum? Let $(, \quad)$ denote the Hilbert symbol. For any quadratic space $V$, let $\alpha V$ denote the same space, but with the bilinear form multiplied by the scalar $\alpha$. Then we have

$$
\begin{aligned}
\operatorname{disc}(\alpha V) & =\alpha^{\operatorname{dim} V} \operatorname{disc} V \\
\operatorname{Hasse}(\alpha V) & =(\alpha, \alpha)^{\left(\frac{\operatorname{dim} V}{2}\right)} \cdot(\alpha, \operatorname{disc} V)^{\operatorname{dim} V-1} \cdot \operatorname{Hasse}(V) \\
\operatorname{disc}\left(V_{1} \oplus V_{2}\right) & =\operatorname{disc}\left(V_{1}\right) \cdot \operatorname{disc}\left(V_{2}\right) \\
\operatorname{Hasse}\left(V_{1} \oplus V_{2}\right) & =\operatorname{Hasse}\left(V_{1}\right) \cdot \operatorname{Hasse}\left(V_{2}\right) \cdot\left(\operatorname{disc} V_{1}, \operatorname{disc} V_{2}\right) .
\end{aligned}
$$

Consider $V=\alpha V_{0} \oplus V_{1}$, where $\alpha$ is a scalar and $V_{0}$ is as above. Then

$$
\begin{aligned}
\operatorname{dim}(V) & =4+\operatorname{dim} V_{1} \\
\operatorname{disc}(V) & =\operatorname{disc}\left(V_{1}\right) \\
\operatorname{Hasse}(V) & =-\operatorname{Hasse}\left(V_{1}\right) .
\end{aligned}
$$

By varying $V_{1}$, we get the following possibilities for $V$ :

\begin{tabular}{ccc}
$\operatorname{dim} V$ & $\operatorname{disc} V$ & Hasse $V$ \\
\hline 4 & 1 & -1 \\
5 & anything & -1 \\
6 & -1 & -1 \\
6 & not -1 & anything \\
$\geq 7$ & anything & anything.
\end{tabular}

Thus, this method finds singular anisotropic elements in every form of $\mathfrak{s o}(n)(n \geq 4)$ with the exception of the isotropic forms of $\mathfrak{s o}(4)$ and the split forms of $\mathfrak{s o}(5)$ and $\mathfrak{s o}(6)$. By a low-dimensional coincidence, these last two are isomorphic to the split forms of $\mathfrak{s p}(4)$ and $\mathfrak{s l}(4)$, respectively. We already know that the former has singular anisotropic elements, and the latter does not. 
3.3. The exceptional group $\mathbf{G}_{2}$. Assume that $p>7$. Let $G$ be the Chevalley group of type $\mathrm{G}_{2}$. A complete set of minimal K-types of $G$ is given in [25], as well as a 7-dimensional representation of $\mathfrak{g}$ (due to Seligman [34]), which we use below.

Let $\varepsilon$ be a nonsquare unit in $F$. Let

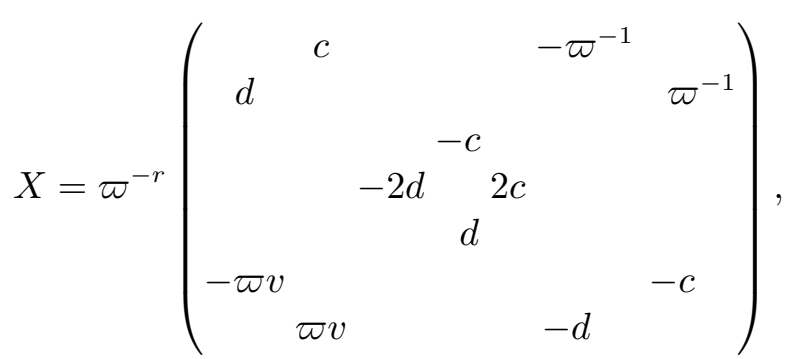

where $c, d$, and $v$ are elements of $\mathcal{O}^{\times}$taken $\bmod \varpi \mathcal{O}, v=c d$ or $9 c d$, and $-v$ is not a square $\bmod \varpi \mathcal{O}$. This falls in case (1.10f.4) of [25]. (Moy suggested using a particular element of this family.) Then $C_{\mathfrak{g}}(X)$ is isomorphic to the anisotropic form of $\mathfrak{u}(2)$.

\section{References}

[1] J.D. Adler and S. DeBacker, Moy-Prasad filtrations and harmonic analysis, preprint.

[2] J.D. Adler and A. Roche, An intertwining result for p-adic groups, preprint, 1997.

[3] C.J. Bushnell and P. Kutzko, The admissible dual of $G L_{N}$ via restriction to compact open subgroups, Ann. of Math. Studies, Vol. 129, Princeton University Press, 1993.

[4] Smooth representations of reductive p-adic groups: Structure theory via types, preprint, 1996.

[5] H. Carayol, Représentations cuspidales du groupe linéaire, Ann. Sci. Éc. Norm. Sup., 17 (1984), 191-225.

[6] R.W. Carter, Simple groups of Lie type, Wiley, 1972.

[7] Finite groups of Lie type: Conjugacy classes and complex characters, Wiley, 1985.

[8] W. Casselman, Introduction to the theory of admissible representations of $p$-adic reductive groups, preprint.

[9] L. Corwin, Representations of division algebras over local fields, Adv. in Math., 13 (1974), 259-267.

[10] P. Gérardin, Construction de séries discrètes p-adiques, Lecture Notes in Mathematics, Vol. 462, Springer, 1975.

[11] W Weil representations associated to finite fields, J. Algebra, 46 (1977), 54101.

[12] R. Howe, On the character of Weil's representation, Trans. Amer. Math. Soc., 177 (1973), 287-298.

[13] - Tamely ramified supercuspidal representations of $G L_{n}$, Pacific J. Math., 73(2) (1977), 437-460. 
[14] R. Howe (with the collaboration of A. Moy), Harish-Chandra homomorphisms for p-adic groups, CBMS Regional Conference Series in Mathematics, Vol. 59, Amer. Math. Soc., 1985.

[15] R. Howe and A. Moy, Minimal K-types for $G L_{n}$ over a p-adic field, Asterisque, 171-172 (1989), 257-273.

[16] Hecke algebra isomorphisms for $G L_{n}$ over a $p$-adic field, J. Algebra, 131(2) (1990), 388-424.

[17] D. Jabon, The supercuspidal representations of $U(2,1)$ and $G S p_{4}$, Ph.D. thesis, University of Chicago, August 1989.

[18] N. Jacobson, Basic algebra II, 2nd ed., W.H. Freeman, 1989.

[19] J. Kim, Hecke algebras of symplectic groups over p-adic fields and supercuspidal representations, Ph.D. thesis, Yale University, 1997.

[20] P. Kutzko, Character formulas for supercuspidal representations of $G L_{\ell}, \ell$ a prime, Amer. J. Math., 109 (1987), 201-222.

[21] L. Morris, Some tamely ramified supercuspidal representations of symplectic groups, Proc. London Math. Soc., 63(3) (1990), 519-551.

[22] - Tamely ramified supercuspidal representations of classical groups I: Filtrations, Ann. Sci. Éc. Norm. Sup., 24 (1991), 705-738.

[23] - Tamely ramified supercuspidal representations of classical groups II: Representation theory, Ann. Sci. Éc. Norm. Sup., 25 (1992), 233-274.

[24] A. Moy, Representations of $U(2,1)$ over a p-adic field, J. Reine Angew. Math., 372 (1986), 178-208.

[25] Minimal K-types for $G_{2}$ over a p-adic field, Trans. Amer. Math. Soc., 305(2) (1988), 517-529.

[26] , Representations of GSp(4) over a p-adic field I and II, Compositio Mathematica, 66 (1988), 237-328.

[27] A. Moy and G. Prasad, Unrefined minimal K-types for p-adic groups, Inv. Math., 116 (1994), 393-408.

[28] Jacquet functors and unrefined minimal K-types, Comment. Math. Helvetici, 71 (1996), 98-121.

[29] F. Murnaghan, private communication.

[30] S.-Y. Pan and J.-K. Yu, Unrefined minimal K-types for p-adic classical groups, preprint, 1997.

[31] G. Prasad and M.S. Raghunathan, Topological central extensions of semi-simple groups over local fields I, Ann. of Math., 119 (1984), 143-201.

[32] A. Roche, Types and Hecke algebras for principal series representations of split reductive p-adic groups, Ph.D. thesis, University of Chicago, 1996.

[33] G. Rousseau, Immeubles des groupes réductifs sur les corps locaux, Ph.D. thesis, Paris XI, 1977.

[34] G. Seligman, On automorphisms of Lie algebras of classical type II, Trans. Amer. Math. Soc., 94 (1960), 452-482.

[35] T. Springer and R. Steinberg, Conjugacy classes, Seminar on Algebraic Groups and Related Finite Groups (A. Borel and R. Carter et al, eds.), Lecture Notes in Mathematics, Vol. 131, Springer, (1970), 167-266. 
[36] R. Steinberg, Torsion in reductive groups, Adv. in Math., 15 (1975), 63-92.

Received September 12, 1996 and revised August 19, 1997.

UNIVERSITY OF TORONTO

Toronto, Ontario

Canada M5S 3G3

E-mail address: adler@math.toronto.edu 